\title{
Algorithms for a Generalized Multipolar Neutrosophic Soft Set with Information Measures to Solve Medical Diagnoses and Decision-Making Problems
}

\author{
Rana Muhammad Zulqarnain $\left(\mathbb{D},{ }^{1}\right.$ Harish Garg $\mathbb{D D}^{2}{ }^{2}$ Imran Siddique, ${ }^{3}$ Rifaqat Ali, \\ Abdelaziz Alsubie, ${ }^{5}$ Nawaf N. Hamadneh $\left(\mathbb{1},{ }^{5}\right.$ and Ilyas Khan $\mathbb{1}^{6}$ \\ ${ }^{1}$ Department of Mathematics, University of Management and Technology, Lahore, Sialkot Campus, Pakistan \\ ${ }^{2}$ School of Mathematics, Thapar Institute of Engineering \& Technology (Deemed University), Patiala 147004, Punjab, India \\ ${ }^{3}$ Department of Mathematics, University of Management and Technology, Lahore 54770, Pakistan \\ ${ }^{4}$ Department of Mathematics, College of Science and Arts, King Khalid University, Muhayil, 61413 Abha, Saudi Arabia \\ ${ }^{5}$ Department of Basic Sciences, College of Science and Theoretical Studies, Saudi Electronic University, Riyadh, Saudi Arabia \\ ${ }^{6}$ Faculty of Mathematics and Statistics, Ton Duc Thang University, Ho Chi Minh City, Vietnam \\ Correspondence should be addressed to Ilyas Khan; ilyaskhan@tdtu.edu.vn
}

Received 21 October 2020; Revised 16 March 2021; Accepted 20 March 2021; Published 8 May 2021

Academic Editor: Stanislaw Migorski

Copyright $\odot 2021$ Rana Muhammad Zulqarnain et al. This is an open access article distributed under the Creative Commons Attribution License, which permits unrestricted use, distribution, and reproduction in any medium, provided the original work is properly cited.

\begin{abstract}
The aim of this paper is to propose the generalized version of the multipolar neutrosophic soft set with operations and basic properties. Here, we define the AND, OR, Truth-Favorite, and False-Favorite operators along with their properties. Also, we define the necessity and possibility of operations for them. Later on, to extend it to solve the decision-making problems, we define some information measures such as distance, similarity, and correlation coefficient for the generalized multipolar neutrosophic soft set. Several desirable properties and their relationship between them are derived. Finally, based on these information measures, a decision-making algorithm is stated under the neutrosophic environment to tackle the uncertain and vague information. The applicability of the proposed algorithm is demonstrated through a case study of the medical-diagnosis and the decision-making problems. A comparative analysis with several existing studies reveals the effectiveness of the approach.
\end{abstract}

\section{Introduction}

Uncertainty plays a dynamic part in numerous fields of life such as modeling, medical, and engineering fields. However, a general question of how we can express and use the uncertainty concept in mathematical modeling is raised. A lot of researchers in the world proposed and recommended different approaches to use uncertainty theory. First of all, Zadeh developed the notion of fuzzy sets [1] to solve those problems which contain uncertainty and vagueness. It is observed that in some cases circumstances cannot be handled by fuzzy sets; to overcome such types of situations, Turksen [2] gave the idea of the interval-valued fuzzy set (IVFS). In some cases, we must deliberate membership unbiassed as the nonmembership values for the suitable representation of an object in uncertain and indeterminate conditions that could not be handled by fuzzy sets or by IVFS. To overcome these difficulties, Atanassov presented the notion of intuitionistic fuzzy sets (IFSs) in [3]. The theory that was presented by Atanassov only deals with the insufficient data considering both membership and nonmembership values; however, the IFSs theory cannot handle the overall incompatible as well as imprecise information. To address such incompatible as well as imprecise records, the idea of the neutrosophic set (NS) was developed by Smarandache [4]. A general mathematical tool was proposed by Molodtsov [5] to deal with indeterminate, fuzzy, and not clearly defined substances known as a soft set (SS). Maji et al. [6] extended the work on SS and defined some operations and their properties. Maji et al. [7] utilized the SS theory for 
decision-making. Ali et al. [8] revised the Maji approach to SS and developed some new operations with their properties. De Morgan's Law on SS theory was proved in [9] by using different operators.

Maji [10] offered the idea of a neutrosophic soft set (NSS) with necessary operations and properties. The idea of the possibility NSS was developed by Karaaslan [11] and introduced a possibility of neutrosophic soft decision-making method to solve those problems which contain uncertainty based on And-product. Broumi [12] developed the generalized NSS with some operations and properties and used the proposed concept for decision-making. To solve MCDM problems with single-valued neutrosophic numbers (SVNNs) presented by Deli and Subas in [13], they constructed the concept of cut sets of SVNNs. On the basis of the correlation of IFS, the term correlation coefficient (CC) of SVNSs [14] was introduced. Ye [15] presented the simplified NSs introduced with some operational laws and aggregation operators such as weighted arithmetic and weighted geometric average operators. Therein, a multicriteria decisionmaking (MCDM) method was constructed based on proposed aggregation operators. Masooma et al. [16] progressed a new concept by combining the multipolar fuzzy set and neutrosophic set, which is known as the multipolar neutrosophic set. They also established various characterizations and operations with examples. Dey et al. [17] developed the grey relational projection method based on NSS to solve MADM complications. Pramanik et al. [18] extended the VIKOR technique to solve MAGDM problems under a bipolar neutrosophic set environment. Pramanik et al. [19] established the TOPSIS technique to solve MADM problems utilizing single-valued neutrosophic soft expert sets. Pramanik et al. [20] developed three different hybrid projection measures projection, bidirectional projection, and hybrid projection measures between bipolar neutrosophic sets.

Peng et al. [21] established the probability multivalued neutrosophic set by combining the multivalued neutrosophic set and probability distribution and used it for decision-making problems. Kamal et al. [22] proposed the idea of mPNSS with some important operations and properties; they also used the developed technique for decision-making. Garg [23] developed the MCDM method based on weighted cosine similarity measures under an intuitionistic fuzzy environment and used the proposed technique for pattern recognition and medical diagnoses. To measure the relative strength of IFS, Garg and Kumar [24] presented some new similarity measures. They also formulated a connection number for set pair analysis (SPA) and developed some new similarity measures and weighted similarity measures based on defined SPA. Garg and Rani [25] extended the IFS technique to complex intuitionistic fuzzy sets (CIFS) and developed the correlation and weighted correlation coefficient under the CIFS environment. To measure the relation between two Pythagorean fuzzy sets (PFS), Garg [26] proposed a novel CC and WCC and presented the numerical examples of pattern recognition and medical diagnoses to verify the validity of the proposed measures. Zulqarnain et al. [27] developed the aggregation operators for Pythagorean fuzzy soft sets and proposed a decision-making methodology using their developed aggregation operators. They also utilized their established decision-making technique for the selection of suppliers in green supply chain management. Zulqarnain et al. [28] extended the TOPSIS technique under Pythagorean fuzzy soft environment. Nguyen et al. [29] defined some similarity measures for PFS by using the exponential function for the membership and nonmembership degrees with its several properties and relations. Peng and Garg [30] presented some diverse types of similarity measures for PFS with multiple parameters. Wang and $\mathrm{Li}$ [31] introduced Pythagorean fuzzy interaction power Bonferroni mean (PBM) operators for solving MADM issues. Wang et al. [32] proposed the Pythagorean fuzzy interactive Hamacher power aggregation operators for assessment of express service quality with entropy weight. Saeed et al. [33] established the concept of mPNSS with its properties and operators; they also developed the distance-based similarity measures and used the proposed similarity measures for decision-making and medical diagnoses.

Gerstenkorn and Mafiko [34] proposed the functional measuring of the interrelation of IFSs, which is known nowadays as correlation, and developed its coefficient with properties. To measure the interrelation of fuzzy numbers, $\mathrm{Yu}$ [35] established the CC of fuzzy numbers. Evaluating the CC for fuzzy data had been developed by Chiang and Lin [36]. Hung and $\mathrm{Wu}$ [37] proposed the centroid method to calculate the CC of IFSs and extended the proposed method to interval-valued intuitionistic fuzzy sets (IVIFSs). Hong [38] and Mitchell [39] also established the CC for IFSs and IVIFSs, respectively. Ye [40] extended the work on IFSs and developed the CC of a single-valued neutrosophic set and developed a decision-making method for similarity measure. Xue et al. [41] developed the CC on a single-valued neutrosophic set and proposed a decision-making method for pattern recognition. Zulqarnain et al. [42] utilized the neutrosophic TOPSIS in the production industry for supplier selection. Garg and Arora [43] introduced the correlation measures on intuitionistic fuzzy soft sets and constructed the TOPSIS technique on developed correlation measures. In Iryna et al.'s work [44], an algorithm has been proposed to handle uncertainty in fault diagnoses by using single-valued neutrosophic sets. Faruk [45] established CC between possibility NSS and proved some properties. He also developed CC for a single-valued neutrosophic refined soft set, and it was used for clustering analysis [46]. A correlation measure of neutrosophic refined sets has been developed, which is the extension of the correlation measure of neutrosophic sets and intuitionistic fuzzy multisets [47].

In this era, professionals consider that the real life is moving in the direction of multipolarity. Thus, it projects as no surprise that multipolarity in information performs a significant part in flourishing numerous fields of science as well as technology. In neurobiology, multipolar neurons in the brain gather a good deal of information from other neurons. In information technology, multipolar technology could be used to control extensive structures. The motivation of the present research is extended and hybrid work is given step by step in the complete article. We demonstrate that 
different hybrid structures containing fuzzy sets are converted into the special privilege of mPNSS under whatsoever appropriate circumstances. The concept of a neutrosophic environment to a multipolar neutrosophic soft set is novel. We tend to discuss the effectiveness, flexibility, quality, and favorable position of our planned work and algorithms. The present research will be the most generalized form and is used to assemble data in considerable and appropriate medical, engineering, artificial intelligence, agriculture, and other everyday life complications. In the future, the present work might be gone competently for other approaches and different types of hybrid structures.

The remainder of the paper is organized as follows: in Section 2, we recollected some basic definitions which are used in the following sequel such as NS, SS, NSS, and multipolar neutrosophic set. In Section 3, we proposed the generalized version of mPNSS with its properties and operations, and we also developed the Truth-Favorite, FalseFavorite, AND, and OR operators in this section. In Section 4, distance-based similarity measures have been developed by using Hamming distance and Euclidean distance between two generalized multipolar neutrosophic soft sets (GmPNSS). In Section 5, the idea of CC and WCC with their properties has been established. Finally, we use the developed distance-based similarity measures and CC for medical diagnoses and decision-making in Section 6. We also present the comparative study of our proposed similarity measures and CC with some already existing techniques in Section 7.

\section{Preliminaries}

In this section, we recollect some basic concepts such as neutrosophic set, soft set, neutrosophic soft set, and m-polar neutrosophic soft set, which are used in the following sequel.

\section{Definition 1 (see [4]).}

Let $\mathcal{U}$ be a universe and let $\mathscr{A}$ be an NS on $\mathcal{U}$ defined as $\mathscr{A}=\left\{u,\left(u_{\mathscr{A}}(u), v_{\mathscr{A}}(u), w_{\mathscr{A}}(u)\right): u \in \mathscr{U}\right\}$, where $u, v, w: \mathscr{U}$ $\longrightarrow 0^{-}, 1^{+}$and $0^{-} \leq u_{\mathscr{A}}(u)+v_{\mathscr{A}}(u)+w_{\mathscr{A}}(u) \leq 3^{+}$.

Definition 2 (see [5]).

Let $\mathscr{U}$ be the universal set and let $\mathscr{E}$ be the set of attributes concerning $\mathcal{U}$. Let $\mathscr{P}(\mathscr{U})$ be the power set of $\mathcal{U}$ and $\mathscr{A} \subseteq \mathscr{E}$. A pair $(\mathscr{F}, \mathscr{A})$ is called a soft set over $\mathcal{U}$ and its mapping is given as

$$
\mathscr{F}: \mathscr{A} \longrightarrow \mathscr{P}(\mathscr{U}) .
$$

It is also defined as

$$
(\mathscr{F}, \mathscr{A})=\{\mathscr{F}(e) \in \mathscr{P}(\mathscr{U}): e \in \mathscr{E}, \mathscr{F}(e)=\varnothing \text { if } e \notin \mathscr{A}\} .
$$

\section{Definition 3 (see [10]).}

Let $\mathscr{U}$ be the universal set and let $\mathscr{E}$ be the set of attributes concerning $\mathscr{U}$. Let $\mathscr{P}(\mathscr{U})$ be the set of neutrosophic values of $\mathscr{U}$ and $\mathscr{A} \subseteq \mathscr{E}$. A pair $(\mathscr{F}, \mathscr{A})$ is called a neutrosophic soft set over $\mathcal{U}$ and its mapping is given as

$$
\mathscr{F}: \mathscr{A} \longrightarrow \mathscr{P}(\mathscr{U})
$$

Definition 4 (see [16]).

Let $\mathcal{U}$ be the universal set and let $\mathscr{E}$ be the set of attributes concerning $\mathcal{U}$; then $\mathscr{F}_{\mathscr{G}}$ is said to be a multipolar neutrosophic set if $\mathscr{F}_{\mathscr{G}}=\left\{u,\left(s_{i} \bullet u_{e}(u), s_{i} \bullet v_{e}(u), s_{i}\right.\right.$ - $\left.\left.w_{e}(u)\right): u \in U, e \in E, i=1,2,3, \ldots, m\right\}$ where $s_{i} \bullet u_{\mathscr{g}}, s_{i} \bullet v_{\mathscr{g}}, s_{i} \bullet w_{\mathscr{E}}: \mathcal{U} \longrightarrow[0,1]$, and $0 \leq s_{i} \bullet u_{\mathscr{E}}(u)+$ $s_{i} \bullet v_{\mathscr{E}}(u)+s_{i} \bullet w_{\mathscr{E}}(u) \leq 3 ; i=1,2,3, \ldots, m . u_{e}, v_{e}$, and $w_{e}$ represent the truth, indeterminacy, and falsity of the considered alternative.

\section{Generalized Multipolar Neutrosophic Soft Set (GmPNSS) with Operators and Properties}

In this section, we develop the concept of GmPNSS and introduce aggregate operators on GmPNSS with their properties.

Definition 5. Let $\mathcal{U}$ and $E$ be universal and set of attributes, respectively, and $\mathscr{A} \subseteq E$, if there exists a mapping $\Phi$ such that

$$
\Phi: \mathscr{A} \longrightarrow \text { GmPNSS }^{\mathscr{U}} \text {, }
$$

then $(\Phi, \mathscr{A})$ is called GmPNSS over $\mathcal{U}$ defined as follows:

$$
Y_{k}=(\Phi, \mathscr{A})=\left\{\left(e,\left(u, \Phi_{\mathscr{A}(e)}(u)\right)\right): e \in E, u \in \mathcal{U}\right\},
$$

where $\Phi_{\mathscr{A}}(e)=\left\{u,\left(s_{i} \bullet u_{\mathscr{A}(e)}(u), s_{i} \bullet v_{\mathscr{A}(e)}(u), s_{i} \bullet w_{\mathscr{A}(e)}\right.\right.$ $(u)): u \in \mathcal{U}, e \in E ; i \in 1,2,3, \ldots, m\}$, and $0 \leq s_{i} \bullet u_{\mathscr{A}(e)}$ $(u)+s_{i} \bullet v_{\mathscr{A}(e)}(u)+s_{i} \bullet w_{\mathscr{A}(e)}(u) \leq 3$ for all $i \in 1,2,3, \ldots, m$; $e \in E$ and $u \in \mathcal{U}$.

Definition 6. Let $\Upsilon_{\mathscr{A}}$ and $\Upsilon_{B}$ be two GmPNSS over $\mathcal{U}$; then $\Upsilon_{\mathscr{A}}$ is called a multipolar neutrosophic soft subset of $\Upsilon_{B}$, if

$$
\begin{gathered}
s_{i} \bullet u_{\mathscr{A}(e)}(u) \leq s_{i} \bullet u_{B(e)}(u), \\
s_{i} \bullet v_{\mathscr{A}(e)}(u) \leq s_{i} \bullet v_{B(e)}(u), \\
s_{i} \bullet w_{\mathscr{A}(e)}(u) \geq s_{i} \bullet w_{B(e)}(u),
\end{gathered}
$$

for all $i \in 1,2,3, \ldots, m ; e \in E$ and $u \in \mathcal{U}$.

Definition 7. Let $\Upsilon_{\mathscr{A}}$ and $\Upsilon_{B}$ be two GmPNSS over $\mathcal{U}$, then $\Upsilon_{\mathscr{A}}=\Upsilon_{B}$, if

$$
\begin{gathered}
s_{i} \bullet u_{\mathscr{A}(e)}(u) \leq s_{i} \bullet u_{B(e)}(u), \\
s_{i} \bullet u_{B(e)}(u) \leq s_{i} \bullet u_{\mathscr{A}(e)}(u), \\
s_{i} \bullet v_{\mathscr{A}(e)}(u) \geq s_{i} \bullet v_{B(e)}(u), \\
s_{i} \bullet v_{B(e)}(u) \geq s_{i} \bullet v_{\mathscr{A}(e)}(u), \\
s_{i} \bullet w_{\mathscr{A}(e)}(u) \geq s_{i} \bullet w_{B(e)}(u), \\
s_{i} \bullet w_{B(e)}(u) \geq s_{i} \bullet w_{\mathscr{A}(e)}(u),
\end{gathered}
$$

for all $i \in 1,2,3, \ldots, m ; e \in E$ and $u \in \mathcal{U}$.

Definition 8. Let $\mathscr{F}_{\mathscr{A}}$ be a GmPNSS over $\mathcal{U}$, then empty GmPNSS can be represented as $\mathscr{F}_{0}$ and defined as follows: 


$$
\mathscr{F}_{\check{0}}=\{e,(u,(0,1,1),(0,1,1), \ldots,(0,1,1)): e \in E, u \in \mathcal{U}\} .
$$

Definition 9. Let $\mathscr{F}_{\mathscr{A}}$ be a GmPNSS over $\mathcal{U}$, then universal GmPNSS can be represented as $\mathscr{F}_{\check{E}}$ and defined as follows:

$$
\mathscr{F}_{\check{E}}=\{e,(u,(1,0,0),(1,0,0), \ldots,(1,0,0)): e \in E, u \in \mathscr{U}\}
$$

Definition 10. Let $\mathscr{F}_{\mathscr{A}}$ be a GmPNSS over $\mathcal{U}$, then the complement of GmPNSS is defined as follows:

$$
\mathscr{F}_{\mathscr{A}}^{c}(e)=\left\{\left(e,\left(u, s_{i} \bullet w_{\mathscr{A}(e)}(u), 1-s_{i} \bullet v_{\mathscr{A}(e)}(u), s_{i} \bullet u_{\mathscr{A}(e)}(u)\right)\right): u \in \mathscr{U}\right\},
$$

for all $i \in 1,2,3, \ldots, m$; $e \in E$ and $u \in \mathcal{U}$.

(3) $\left(\mathscr{F}_{\check{E}}\right)^{c}=\mathscr{F}_{\check{0}}$

Proposition 1. If $\mathscr{F}_{\mathscr{A}}$ is a GmPNSS, then

(1) $\left(\mathscr{F}_{\mathscr{A}}^{c}\right)^{c}=\mathscr{F}_{\mathscr{A}}$

Proof. Let

(2) $\left(\mathscr{F}_{\check{0}}\right)^{c}=\mathscr{F}_{\breve{E}}$

$$
\mathscr{F}_{\mathscr{A}}(e)=\left\{\left(e,\left(u,\left(s_{i} \bullet u_{\mathscr{A}(e)}(u), s_{i} \bullet v_{\mathscr{A}(e)}(u), s_{i} \bullet w_{\mathscr{A} e}(u)\right)\right)\right): u \in U, e \in E ; i \in 1,2,3, \ldots, m\right\} .
$$

Then, by using Definition 10, we get

$$
\mathscr{F}_{\mathscr{A}}^{c}(e)=\left\{\left(e,\left(u,\left(s_{i} \bullet w_{\mathscr{A}(e)}(u), 1-s_{i} \bullet v_{\mathscr{A}(e)}(u), s_{i} \bullet u_{\mathscr{A}(e)}(u)\right)\right)\right): u \in U, e \in E ; i \in 1,2,3, \ldots, m\right\} .
$$

Again, by using Definition 10,

$$
\begin{aligned}
& \left(\mathscr{F}_{\mathscr{A}}^{c}(e)\right)^{c}=\left\{\left(e,\left(u,\left(s_{i} \bullet u_{\mathscr{A}(e)}(u), 1-\left(1-s_{i} \bullet v_{\mathscr{A}(e)}(u)\right), s_{i} \bullet w_{\mathscr{A}(e)}(u)\right)\right)\right): u \in U, e \in E ; i \in 1,2,3, \ldots, m\right\}, \\
& \left(\mathscr{F}_{\mathscr{A}}^{c}(e)\right)^{c}=\left\{\left(e,\left(u,\left(s_{i} \bullet u_{\mathscr{A}(e)}(u), s_{i} \bullet v_{\mathscr{A}(e)}(u), s_{i} \bullet w_{\mathscr{A}(e)}(u)\right)\right)\right): u \in U, e \in E ; i \in 1,2,3, \ldots, m\right\} \\
& \left(\mathscr{F}_{\mathscr{A}}^{c}(e)\right)^{c}=\mathscr{F}_{\mathscr{A}}(e) .
\end{aligned}
$$

Proof. Let $\mathscr{F}_{\check{0}}$ be an empty GmPNSS over $\mathcal{U}$.

$$
\mathscr{F}_{\check{0}}=\{e,(u,(0,1,1),(0,1,1), \ldots,(0,1,1)): e \in E, u \in \mathcal{U}\} .
$$


Utilizing Definition 10,

$$
\begin{aligned}
& \left(\mathscr{F}_{0}\right)^{c}=\{e,(u,(1,0,0),(1,0,0), \ldots,(1,0,0)): e \in E, u \in \mathcal{U}\}, \\
& \left(\mathscr{F}_{\check{0}}\right)^{c}=\mathscr{F}_{\check{E}} .
\end{aligned}
$$

Similarly, we can prove 3 .

Definition 11. Let $\mathscr{F}_{\mathscr{A}(e)}$ and $\mathscr{G}_{B(e)}$ be two GmPNSS over $\mathscr{U}$. Then,

$$
\mathscr{F}_{\mathscr{A}(e)} \cup \mathscr{G}_{B(e)}=\left\{\left(e,\left(u,\left(\begin{array}{c}
\max \left\{s_{i} \bullet u_{\mathscr{A}(e)}(u), s_{i} \bullet u_{B(e)}(u)\right\} \\
\min \left\{s_{i} \bullet v_{\mathscr{A}(e)}(u), s_{i} \bullet v_{B(e)}(u)\right\} \\
\min \left\{s_{i} \bullet w_{\mathscr{A}(e)}(u), s_{i} \bullet w_{B(e)}(u)\right\}
\end{array}\right)\right): u \in U, e \in E ; i \in 1,2,3, \ldots, m\right\} .\right.
$$

Proposition 2. Let $\mathscr{F}_{\check{A}}, \mathscr{G}_{\check{B}}$, and $\mathscr{H}_{\check{C}}$ be GmPNSS over $\mathcal{U}$. Then,

(1) $\mathscr{F}_{\check{A}} \cup \mathscr{F}_{\check{A}}=\mathscr{F}_{\check{A}}$

(2) $\mathscr{F}_{\check{A}} \cup \mathscr{F}_{\check{0}}=\mathscr{F}_{\check{A}}$
(3) $\mathscr{F}_{\check{A}} \cup \mathscr{G}_{\check{B}}=\mathscr{G}_{\check{B}} \cup \mathscr{F}_{\check{A}}$

(4) $\left(\mathscr{F}_{\check{A}} \cup \mathscr{G}_{\check{B}}\right) \cup \mathscr{H}_{\check{C}}=\mathscr{F}_{\check{A}} \cup\left(\mathscr{G}_{\check{B}} \cup \mathscr{H}_{\check{C}}\right)$

$$
\mathscr{F}_{\check{A}}(e)=\left\{\left(e,\left(u,\left(s_{i} \bullet u_{\check{A}(e)}(u), s_{i} \bullet v_{\check{A}(e)}(u), s_{i} \bullet w_{\check{A}(e)}(u)\right)\right)\right): u \in U, e \in E ; i \in 1,2,3, \ldots, m\right\},
$$

be a GmPNSS. Then,

$$
\begin{aligned}
& \mathscr{F}_{\check{A}}(e) \cup \mathscr{F}_{\check{A}}(e)=\left\{\left(e,\left(u,\left(\begin{array}{c}
\max \left\{s_{i} \bullet u_{\check{A}(e)}(u), s_{i} \bullet u_{\check{A}(e)}(u)\right\} \\
\min \left\{s_{i} \bullet v_{\check{A}(e)}(u), s_{i} \bullet v_{\check{A}(e)}(u)\right\} \\
\min \left\{s_{i} \bullet w_{\check{A}(e)}(u), s_{i} \bullet w_{\check{A}(e)}(u)\right\}
\end{array}\right)\right): u \in U, e \in E ; i \in 1,2,3, \ldots, m\right\},\right. \\
& \mathscr{F}_{\check{A}}(e) \cup \mathscr{F}_{\check{A}}(e)=\left\{\left(e,\left(u,\left(s_{i} \bullet u_{\check{A}(e)}(u), s_{i} \bullet v_{\check{A}(e)}(u), s_{i} \bullet w_{\check{A}(e)}(u)\right)\right)\right): u \in U, e \in E ; i \in 1,2,3, \ldots, m\right\}, \\
& \mathscr{F}_{\check{A}} \cup \mathscr{F}_{\check{A}}=\mathscr{F}_{\check{A} .}
\end{aligned}
$$

By using Definition 11, we can easily prove the remaining properties.
Definition 12. Let $\mathscr{F}_{\mathscr{A}(e)}$ and $\mathscr{G}_{B(e)}$ be GmPNSS over $\mathcal{U}$. Then,

$$
\mathscr{F}_{\mathscr{A}(e)} \cap \mathscr{G}_{B(e)}=\left\{\left(e,\left(u,\left(\begin{array}{c}
\min \left\{s_{i} \bullet u_{\mathscr{A}(e)}(u), s_{i} \bullet u_{B(e)}(u)\right\} \\
\max \left\{s_{i} \bullet v_{\mathscr{A}(e)}(u), s_{i} \bullet v_{B(e)}(u)\right\} \\
\max \left\{s_{i} \bullet w_{\mathscr{A}(e)}(u), s_{i} \bullet w_{B(e)}(u)\right\}
\end{array}\right)\right)\right): u \in U, e \in E ; i \in 1,2,3, \ldots, m\right\}
$$

Proposition 3. Let $\mathscr{F}_{\check{A}}, \mathscr{G}_{\check{B}}$, and $\mathscr{H}_{\check{C}}$ be GmPNSS over $\mathcal{U}$.

Then,

$$
\text { (1) } \mathscr{F}_{\check{A}} \cap \mathscr{F}_{\check{A}}=\mathscr{F}_{\check{A}}
$$

(2) $\mathscr{F}_{\check{A}} \cap \mathscr{F}_{\check{0}}=\mathscr{F}_{\check{A}}$

(3) $\mathscr{F}_{\check{A}} \cap \mathscr{F}_{\check{E}}=\mathscr{F}_{\check{E}}$

(4) $\mathscr{F}_{\check{A}} \cap \mathscr{G}_{\check{B}}=\mathscr{G}_{\check{B}} \cap \mathscr{F}_{\check{A}}$ 

(5) $\left(\mathscr{F}_{\check{A}} \cap \mathscr{G}_{\check{B}}\right) \cap \mathscr{H}_{\check{C}}=\mathscr{F}_{\check{A}} \cap\left(\mathscr{G}_{\check{B}} \cap \mathscr{H}_{\check{C}}\right)$
(1) $\left(\mathscr{F}_{\check{A}(e)} \cup \mathscr{G}_{\check{B}(e)}\right)^{C}=\mathscr{F}_{\check{A}(e)}^{C} \cap \mathscr{G}_{\check{B}(e)}^{C}$

(2) $\left(\mathscr{F}_{\check{A}(e)} \cap G_{B(e)}\right)^{C}=\mathscr{F}_{\check{A}(e)}^{C} \cup \mathscr{G}_{\check{B}(e)}^{C}$

Proof. By using Definition 12, the proof is easy.

Proposition 4. Let $\mathscr{F}_{\check{A}}$ and $\mathscr{G}_{\breve{B}}$ be GmPNSS over $\mathcal{U}$. Then,

Proof. We know that

$$
\begin{aligned}
& \mathscr{F}_{\check{A}}(e)=\left\{\left(e,\left(u,\left(s_{i} \bullet u_{\check{A}(e)}(u), s_{i} \bullet v_{\check{A}(e)}(u), s_{i} \bullet w_{\check{A}(e)}(u)\right)\right)\right): u \in U, e \in E ; i \in 1,2,3, \ldots, m\right\}, \\
& \mathscr{G}_{\check{B}}(e)=\left\{\left(e,\left(u,\left(s_{i} \bullet u_{\check{B}(e)}(u), s_{i} \bullet v_{\check{B}(e)}(u), s_{i} \bullet w_{\check{B}(e)}(u)\right)\right)\right): u \in U, e \in E ; i \in 1,2,3, \ldots, m\right\},
\end{aligned}
$$

are two GmPNSS.

By using Definition 11,

$$
\mathscr{F}_{\check{A}}(e) \cup \mathscr{G}_{\check{B}(e)}=\left\{\left(e,\left(u,\left(\begin{array}{c}
\max \left\{s_{i} \bullet u_{\check{A}(e)}(u), s_{i} \bullet u_{B(e)}(u)\right\} \\
\min \left\{s_{i} \bullet v_{\check{A}(e)}(u), s_{i} \bullet v_{B(e)}(u)\right\} \\
\min \left\{s_{i} \bullet w_{\check{A}(e)}(u), s_{i} \bullet w_{B(e)}(u)\right\}
\end{array}\right)\right): u \in U, e \in E ; i \in 1,2,3, \ldots, m\right\} .\right.
$$

Now, by using Definition 10,

$$
\left(\mathscr{F}_{\check{A}}(e) \cup \mathscr{G}_{\check{B}(e)}\right)^{c}=\left\{\left(e,\left(u,\left(\begin{array}{c}
\min \left\{s_{i} \bullet w_{\check{A}(e)}(u), s_{i} \bullet w_{B(e)}(u)\right\} \\
1-\min \left\{s_{i} \bullet v_{\check{A}(e)}(u), s_{i} \bullet v_{B(e)}(u)\right\} \\
\max \left\{s_{i} \bullet u_{\check{A}(e)}(u), s_{i} \bullet u_{B(e)}(u)\right\}
\end{array}\right)\right): u \in U, e \in E ; i \in 1,2,3, \ldots, m\right\} .\right.
$$

Now,

$$
\begin{gathered}
\mathscr{F}_{\check{A}}(e)^{C}=\left\{\left(e,\left(u,\left(s_{i} \bullet w_{\check{A}(e)}(u), 1-s_{i} \bullet v_{\check{A}(e)}(u), s_{i} \bullet u_{\check{A}(e)}(u)\right)\right)\right): u \in U, e \in E ; i \in 1,2,3, \ldots, m\right\}, \\
\mathscr{G}_{\check{B}(e)}^{C}=\left\{\left(e,\left(u,\left(s_{i} \bullet w_{B(e)}(u), 1-s_{i} \bullet v_{B(e)}(u), s_{i} \bullet u_{B(e)}(u)\right)\right)\right): u \in U, e \in E ; i \in 1,2,3, \ldots, m\right\} .
\end{gathered}
$$

By using Definition 12,

$$
\begin{gathered}
\mathscr{F}_{\check{A}}(e)^{C} \cap \mathscr{G}_{\check{B}(e)}^{C}=\left\{\left(e,\left(u,\left(\begin{array}{c}
\min \left\{s_{i} \bullet w_{\check{A}(e)}(u), s_{i} \bullet w_{B(e)}(u)\right\} \\
\max \left\{1-s_{i} \bullet v_{\check{A}(e)}(u), 1-s_{i} \bullet v_{B(e)}(u)\right\} \\
\max \left\{s_{i} \bullet u_{\check{A}(e)}(u), s_{i} \bullet u_{B(e)}(u)\right\}
\end{array}\right)\right)\right): u \in U, e \in E ; i \in 1,2,3, \ldots m\right\}, \\
\mathscr{F}_{\check{A}}(e)^{C} \cap \mathscr{G}_{\check{B}(e)}^{C}=\left\{\left(e,\left(u,\left(\begin{array}{c}
\min \left\{s_{i} \bullet w_{\check{A}(e)}(u), s_{i} \bullet w_{B(e)}(u)\right\} \\
1-\min \left\{s_{i} \bullet v_{\check{A}(e)}(u), s_{i} \bullet v_{B(e)}(u)\right\} \\
\max \left\{s_{i} \bullet u_{\check{A}(e)}(u), s_{i} \bullet u_{B(e)}(u)\right\}
\end{array}\right)\right): u \in U, e \in E ; i \in 1,2,3, \ldots, m\right\} .\right.
\end{gathered}
$$

Hence,

$$
\mathscr{F}_{\check{A}}(e) \cup \mathscr{G}_{\check{B}(e)}{ }^{C}=\mathscr{F}_{\check{A}}(e)^{C} \cap \mathscr{G}_{\breve{B}(e)}^{C} .
$$


Proof. We know that

$$
\begin{aligned}
& \mathscr{F}_{\check{A}}(e)=\left\{\left(e,\left(u,\left(s_{i} \bullet u_{\check{A}(e)}(u), s_{i} \bullet v_{\check{A}(e)}(u), s_{i} \bullet w_{\check{A}(e)}(u)\right)\right)\right): u \in U, e \in E ; i \in 1,2,3, \ldots, m\right\}, \\
& \mathscr{G}_{\check{B}}(e)=\left\{\left(e,\left(u,\left(s_{i} \bullet u_{\breve{B}(e)}(u), s_{i} \bullet v_{\breve{B}(e)}(u), s_{i} \bullet w_{\check{B}(e)}(u)\right)\right)\right): u \in U, e \in E ; i \in 1,2,3, \ldots, m\right\},
\end{aligned}
$$

are two GmPNSS.

Utilizing Definition 12,

$$
\mathscr{F}_{\check{A}(e)} \cap G_{B(e)}=\left\{\left(e,\left(u,\left(\begin{array}{c}
\min \left\{s_{i} \bullet u_{\check{A}(e)}(u), s_{i} \bullet u_{B(e)}(u)\right\} \\
\max \left\{s_{i} \bullet v_{\check{A}(e)}(u), s_{i} \bullet v_{B(e)}(u)\right\} \\
\max \left\{s_{i} \bullet w_{\check{A}(e)}(u), s_{i} \bullet w_{B(e)}(u)\right\}
\end{array}\right)\right): u \in U, e \in E ; i \in 1,2,3, \ldots, m\right\} .\right.
$$

By Definition 10,

$$
\left(\mathscr{F}_{\check{A}(e)} \cap G_{B(e)}\right)^{C}=\left\{\left(e,\left(u,\left(\begin{array}{c}
\max \left\{s_{i} \bullet w_{\check{A}(e)}(u), s_{i} \bullet w_{B(e)}(u)\right\} \\
1-\max \left\{s_{i} \bullet v_{\check{A}(e)}(u), s_{i} \bullet v_{B(e)}(u)\right\} \\
\min \left\{s_{i} \bullet u_{\check{A}(e)}(u), s_{i} \bullet u_{B(e)}(u)\right\}
\end{array}\right)\right): u \in U, e \in E ; i \in 1,2,3, \ldots, m\right\} .\right.
$$

Now,

$$
\begin{aligned}
\mathscr{F}_{\check{A}}(e)^{C} & =\left\{\left(e,\left(u,\left(s_{i} \bullet w_{\check{A}(e)}(u), 1-s_{i} \bullet v_{\check{A}(e)}(u), s_{i} \bullet u_{\check{A}(e)}(u)\right)\right)\right): u \in U, e \in E ; i \in 1,2,3, \ldots, m\right\}, \\
\mathscr{G}_{\breve{B}(e)}^{C} & =\left\{\left(e,\left(u,\left(s_{i} \bullet w_{B(e)}(u), 1-s_{i} \bullet v_{B(e)}(u), s_{i} \bullet u_{B(e)}(u)\right)\right)\right): u \in U, e \in E ; i \in 1,2,3, \ldots, m\right\} .
\end{aligned}
$$

By using Definition 11,

$$
\begin{gathered}
\mathscr{F}_{\check{A}}(e)^{C} \cup \mathscr{G}_{\breve{B}(e)}^{C}=\left\{\left(e,\left(u,\left(\begin{array}{c}
\max \left\{s_{i} \bullet w_{\check{A}(e)}(u), s_{i} \bullet w_{B(e)}(u)\right\} \\
\min \left\{1-s_{i} \bullet v_{\check{A}(e)}(u), 1-s_{i} \bullet v_{B(e)}(u)\right\} \\
\min \left\{s_{i} \bullet u_{\check{A}(e)}(u), s_{i} \bullet u_{B(e)}(u)\right\}
\end{array}\right)\right): u \in U, e \in E ; i \in 1,2,3, \ldots m\right\},\right. \\
\mathscr{F}_{\check{A}}(e)^{C} \cup \mathscr{G}_{\breve{B}(e)}^{C}=\left\{\left(e,\left(u,\left(\begin{array}{c}
\max \left\{s_{i} \bullet w_{\check{A}(e)}(u), s_{i} \bullet w_{B(e)}(u)\right\} \\
1-\max \left\{s_{i} \bullet v_{\check{A}(e)}(u), s_{i} \bullet v_{B(e)}(u)\right\} \\
\min \left\{s_{i} \bullet u_{\check{A}(e)}(u), s_{i} \bullet u_{B(e)}(u)\right\}
\end{array}\right)\right)\right): u \in U, e \in E ; i \in 1,2,3, \ldots, m\right\} .
\end{gathered}
$$

Hence,

$$
\left(\mathscr{F}_{\check{A}(e)} \cap G_{B(e)}\right)^{C}=\mathscr{F}_{\check{A}(e)}^{C} \cup \mathscr{G}_{\breve{B}(e)}^{C} .
$$


Proposition 5. Let $\mathscr{F}_{A(\check{C})}, \mathscr{G}_{B(e)}$, and $\mathscr{H}_{C \check{C}(e)}$ be GmPNSS over U. Then,

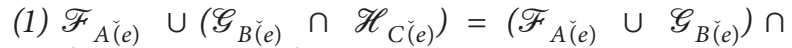
$\left(\mathscr{F}_{A(e)} \cup \mathscr{H}_{C(e)}\right)$

(2) $\mathscr{F}_{A \check{(e)}} \cap\left(\mathscr{G}_{B \check{(e)}} \cup \cup \quad \mathscr{H}_{C \check{C}(e)}\right)=\left(\begin{array}{llll}\mathscr{F}_{A(e)} & \cap & \mathscr{G}_{B(e)}\end{array}\right)$ $\cup\left(\mathscr{F}_{A(e)} \cap \mathscr{H}_{C(e)}\right)$
(3) $\mathscr{F}_{A \check{(}(e)} \cup\left(\mathscr{F}_{A \check{(e)}} \cap \mathscr{G}_{B \check{(\check{(} e)}}\right)=\mathscr{F}_{A \check{(e)}}$

(4) $\mathscr{F}_{A(e)} \cap\left(\mathscr{F}_{A \check{(}(e)} \cup \mathscr{G}_{B(\check{(\check{l})}}\right)=\mathscr{F}_{A(e)}$

Proof. We know that

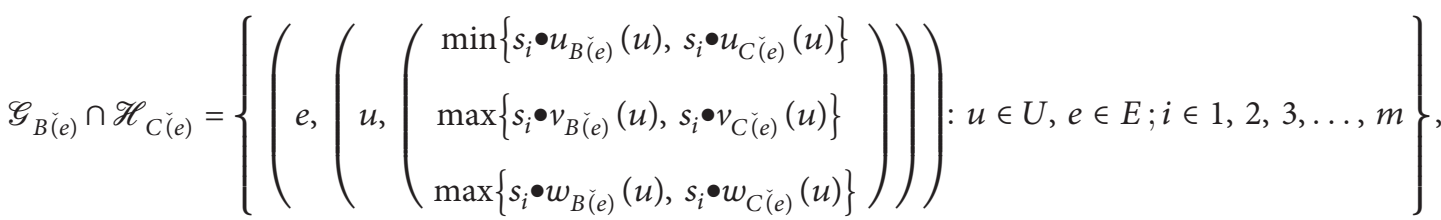

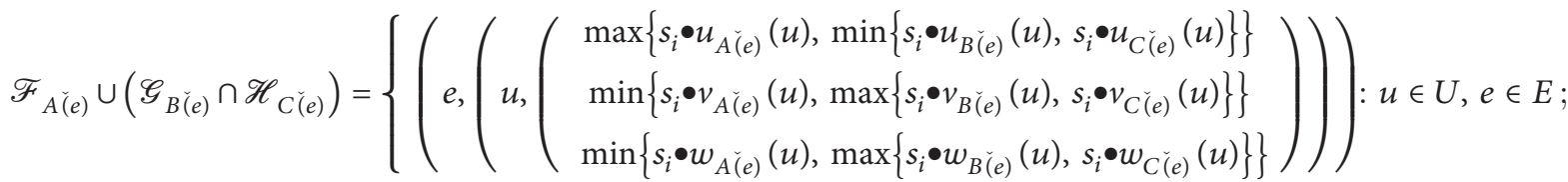

$i \in 1,2,3, \ldots, m\}$,

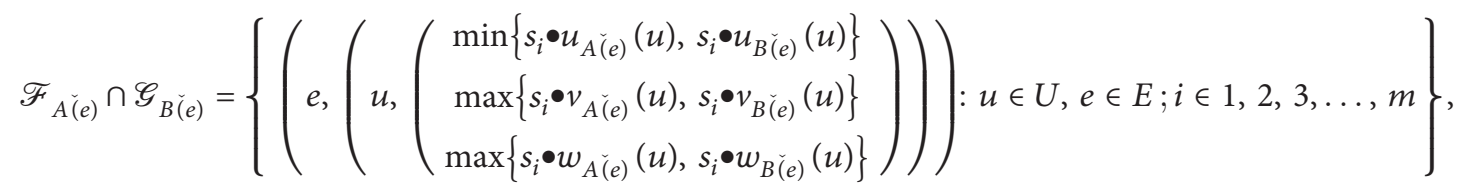

$\mathscr{F}_{A \check{A(e)}} \cap \mathscr{H}_{C \check{C(e)}}=\left\{\left(e,\left(u,\left(\begin{array}{c}\min \left\{s_{i} \bullet u_{A(\check{(e)}}(u), s_{i} \bullet u_{C \check{C}(e)}(u)\right\} \\ \max \left\{s_{i} \bullet v_{A \check{(})}(u), s_{i} \bullet v_{C \check{C}(e)}(u)\right\} \\ \max \left\{s_{i} \bullet w_{A \check{(e)}}(u), s_{i} \bullet w_{C(\check{(e)}}(u)\right\}\end{array}\right)\right): u \in U, e \in E ; i \in 1,2,3, \ldots, m\right\}\right.$,

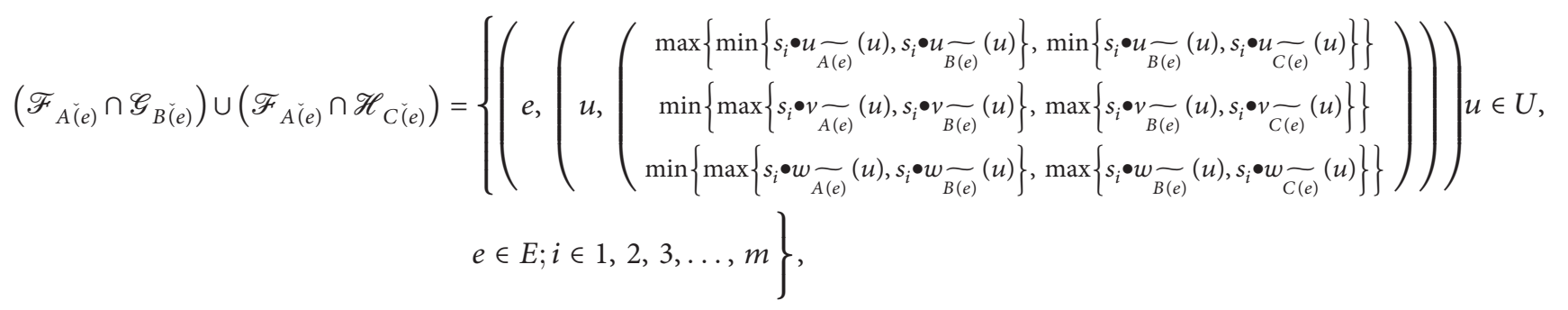

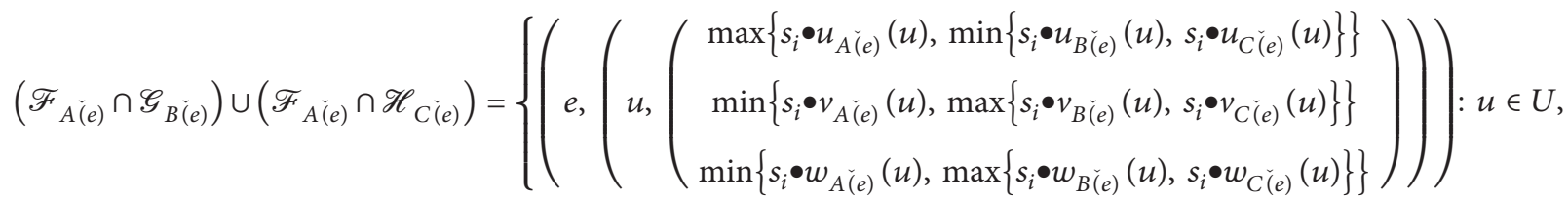

$e \in E ; i \in 1,2,3, \ldots, m\}$. 
Hence,

$$
\mathscr{F}_{A(e)} \cup\left(\mathscr{G}_{B(e)} \cap \mathscr{H}_{C \check{(e)}}\right)=\left(\mathscr{F}_{A \check{(e)}} \cup \mathscr{G}_{B(e)}\right) \cap\left(\mathscr{F}_{A(e)} \cup \mathscr{H}_{C \check{(e)}}\right) .
$$

Similarly, we can prove other results.

Definition 13. Let $\mathscr{F}_{\check{A}}$ and $\mathscr{G}_{\breve{B}}$ be GmPNSS, then their extended union is defined as

$$
\begin{gathered}
u\left(\mathscr{F}_{\check{A}} \cup_{\varepsilon} \mathscr{G}_{\check{B}}\right)= \begin{cases}s_{i} \bullet u_{A(e)}(u), & \text { if } e \in A-B, \\
s_{i} \bullet u_{B(e)}(u), & \text { if } e \in B-A, \\
\max \left\{s_{i} \bullet u_{A(e)}(u), s_{i} \bullet u_{B(e)}(u)\right\}, & \text { if } e \in A \cap B,\end{cases} \\
v\left(\mathscr{F}_{\check{A}} \cup_{\varepsilon} \mathscr{G}_{\check{B}}\right)= \begin{cases}s_{i} \bullet v_{A(e)}(u), & \text { if } e \in A-B, \\
s_{i} \bullet v_{B(e)}(u), & \text { if } e \in B-A, \\
\min \left\{s_{i} \bullet v_{A(e)}(u), s_{i} \bullet v_{B(e)}(u)\right\}, & \text { if } e \in A \cap B,\end{cases} \\
w\left(\mathscr{F}_{\check{A}} \cup_{\varepsilon} \mathscr{G}_{\check{B}}\right)= \begin{cases}s_{i} \bullet w_{A(e)}(u), & \text { if } e \in A-B, \\
s_{i} \bullet w_{B(e)}(u), & \text { if } e \in B-A, \\
\min \left\{s_{i} \bullet w_{A(e)}(u), s_{i} \bullet w_{B(e)}(u)\right\}, & \text { if } e \in A \cap B .\end{cases}
\end{gathered}
$$

Example 1. Assume that $\mathcal{U}=\left\{u_{1}, u_{2}\right\}$ is a universe of discourse and let $E=\left\{x_{1}, x_{2}, x_{3}, x_{4}\right\}$ be a set of attributes and $A=\left\{x_{1}, x_{2}\right\}$ and $B=\left\{x_{2}, x_{3}\right\} \subseteq E$. Consider $\mathscr{F}_{\check{A}(e)}$ and $\mathscr{G}_{\check{B}(e)}$ $\in$ G3-PNSS over $\mathcal{U}$ can be represented as follows:

$$
\begin{gathered}
\mathscr{F}_{\check{A}}=\left\{\begin{array}{c}
\left(x_{1},\left\{u_{1},(.5, .2, .1),(.3, .1, .2),(.6, .7, .8)\right)\right. \\
\left(u_{2},(.2, .3, .1),(.2, .1, .1),(.8, .6, .6)\right) \\
\left(x_{2},\left\{u_{1},(.3, .1, .3),(0, .1, .3),(.5, .3, .5)\right)\right. \\
\left(u_{2},(.2, .2, .5),(.3, .1, .5),(.6, .5, .6)\right)
\end{array}\right\}, \\
\mathscr{G}_{\check{B}}=\left\{\begin{array}{c}
\left(x_{2},\left\{u_{1},(.4, .3, .2),(.2, .3, .4),(.7, .4, .5)\right)\right. \\
\left(u_{2},(.1, .5, .1),(.3, .2, .2),(.5, .7, .4)\right) \\
\left(x_{3},\left\{u_{1},(.2, .3, .2),(.1, .2, .2),(.4, .4, .5)\right)\right. \\
\left(u_{2},(.1, .1, .4),(.3, .3,1),(.5, .3, .1)\right)
\end{array}\right\} .
\end{gathered}
$$

Then,

$$
\mathscr{F}_{\check{A}} \cup_{\varepsilon} \mathscr{G}_{\check{B}}=\left\{\begin{array}{c}
\left(x_{1},\left\{u_{1},(.5, .2, .1),(.3, .1, .2),(.6, .7, .8)\right)\right. \\
\left(u_{2},(.2, .3, .1),(.2, .1, .1),(.8, .6, .6)\right) \\
\left(x_{2},\left\{u_{1},(.4, .1, .2),(.2, .1, .3),(.7, .3, .5)\right)\right. \\
\left(u_{2},(.2, .2, .1),(.3, .1, .2),(.6, .5, .4)\right) \\
\left(x_{3},\left\{u_{1},(.2, .3, .2),(.1, .2, .2),(.4, .4, .5)\right)\right. \\
\left(u_{2},(.1, .1, .4),(.3, .3,1),(.5, .3, .1)\right)
\end{array}\right\} .
$$

Definition 14. Let $\mathscr{F}_{\check{A}}$ and $\mathscr{G}_{\breve{B}}$ be GmPNSS; then their extended union is defined as

$$
\begin{gathered}
u\left(\mathscr{F}_{\check{A}} \cap_{\varepsilon} \mathscr{G}_{\check{B}}\right)= \begin{cases}s_{i} \bullet u_{A(e)}(u), & \text { if } e \in A-B, \\
s_{i} \bullet u_{B(e)}(u), & \text { if } e \in B-A, \\
\min \left\{s_{i} \bullet u_{A(e)}(u), s_{i} \bullet u_{B(e)}(u)\right\}, & \text { if } e \in A \cap B,\end{cases} \\
v\left(\mathscr{F}_{\check{A}} \cap_{\varepsilon} \mathscr{G}_{\check{B}}\right)= \begin{cases}s_{i} \bullet v_{A(e)}(u), & \text { if } e \in A-B, \\
s_{i} \bullet v_{B(e)}(u), & \text { if } e \in B-A, \\
\max \left\{s_{i} \bullet v_{A(e)}(u), s_{i} \bullet v_{B(e)}(u)\right\}, & \text { if } e \in A \cap B,\end{cases}
\end{gathered}
$$




$$
w\left(\mathscr{F}_{\check{A}} \cap_{\varepsilon} \mathscr{G}_{\breve{B}}\right)= \begin{cases}s_{i} \bullet w_{A(e)}(u), & \text { if } e \in A-B, \\ s_{i} \bullet w_{B(e)}(u), & \text { if } e \in B-A, \\ \max \left\{s_{i} \bullet w_{A(e)}(u), s_{i} \bullet w_{B(e)}(u)\right\}, & \text { if } e \in A \cap B .\end{cases}
$$

Definition 15. Let $\mathscr{F}_{\check{A}}$ and $\mathscr{G}_{\check{B}}$ be GmPNSS, then their difference is defined as follows:

$$
\begin{aligned}
\mathscr{F}_{\check{A}} \backslash \mathscr{G}_{\check{B}}= & \left\{\left(e,\left(u, \min \left\{s_{i} \bullet u_{A(e)}(u), s_{i} \bullet u_{B(e)}(u)\right\}, \max \left\{s_{i} \bullet v_{A(e)}(u), 1-s_{i} \bullet v_{B(e)}(u)\right\}, \max \left\{s_{i} \bullet w_{A(e)}(u), s_{i} \bullet w_{B(e)}(u)\right\}\right)\right):\right. \\
& u \in U ; i \in 1,2,3, \ldots, m\} .
\end{aligned}
$$

Definition 16. Let $\mathscr{F}_{\check{A}}$ and $\mathscr{G}_{\check{B}}$ be GmPNSS, then their addition is defined as follows:

$$
\begin{aligned}
\mathscr{F}_{\check{A}}+\mathscr{G}_{\breve{B}}= & \left\{\left(e,\left(u, \min \left\{s_{i} \bullet u_{A(e)}(u)+s_{i} \bullet u_{B(e)}(u), 1\right\}, \min \left\{s_{i} \bullet v_{A(e)}(u)+s_{i} \bullet v_{B}(e)(u), 1\right\}, \min \left\{s_{i} \bullet w_{A}(u)+s_{i} \bullet w_{B}(u), 1\right\}\right):\right.\right. \\
& u \in U ; i \in 1,2,3, \ldots, m)\} .
\end{aligned}
$$

Definition 17. Let $\mathscr{F}_{\check{A}}$ be a GmPNSS, then its scalar multiplication is represented as $\mathscr{F}_{\check{A}}(e)$. $\check{a}$, where $\check{a} \in[0,1]$ and it is defined as follows:

$$
\mathscr{F}_{\check{A}} \cdot \check{a}=\left\{e,\left(u, \min \left\{s_{i} \bullet u_{A(e)}(u) . \check{a}, 1\right\}, \min \left\{s_{i} \bullet v_{A(e)}(u) . \check{a}, 1\right\}, \min \left\{s_{i} \bullet w_{A(e)}(u) . \check{a}, 1\right\}: u \in U\right)\right\} .
$$

Definition 18. Let $\mathscr{F}_{\check{A}}$ be a GmPNSS, then its scalar division is represented as $\mathscr{F}_{\breve{A}} / \check{a}$, where $\check{a} \in[0,1]$ and it is defined as follows:

$$
\mathscr{F}_{\check{A}} / \check{a}=\left\{e,\left(u, \min \left\{s_{i} \bullet \frac{u_{A(e)}(u)}{\check{a}}, 1\right\}, \min \left\{s_{i} \bullet \frac{v_{A(e)}(u)}{\check{a}}, 1\right\}, \min \left\{s_{i} \bullet \frac{w_{A(e)}(u)}{\check{a}}, 1\right\}: u \in U\right)\right\} .
$$

Definition 19. Let $\mathscr{F}_{\check{A}}$ be a GmPNSS over $\mathcal{U}$, then TruthFavorite operator on $\mathscr{F}_{\check{A}}$ can be represented by $\widetilde{\Delta} \mathscr{F}_{\check{A}}$ and it is defined as follows:

$$
\widetilde{\Delta} \mathscr{F}_{\check{A}}=\left(\left\{e, u, \min \left\{s_{i} \bullet u_{A(e)}(u)+s_{i} \bullet v_{A(e)}(u), 1\right\}, 0, s_{i} \bullet w_{A(e)}(u)\right\}: u \in U ; i \in 1,2,3, \ldots, m\right) .
$$


Proposition 6. Let $\mathscr{F}_{\check{A}}$ and $\mathscr{G}_{\check{B}}$ be GmPNSS over $\mathcal{U}$. Then,

(1) $\tilde{\Delta} \widetilde{\Delta} \mathscr{F}_{\check{A}}=\widetilde{\Delta} \mathscr{F}_{\check{A}}$

(2) $\widetilde{\Delta}\left(\mathscr{F}_{\check{A}} \cup \mathscr{G}_{\tilde{B}}\right) \subseteq \widetilde{\Delta} \mathscr{F}_{\check{A}} \cup \widetilde{\Delta} \mathscr{G}_{\check{B}}$

(3) $\widetilde{\Delta}\left(\mathscr{F}_{\check{A}} \cap \mathscr{G}_{\breve{B}}\right) \subseteq \widetilde{\Delta} \mathscr{F}_{\check{A}} \cap \widetilde{\Delta} \mathscr{G}_{\check{B}}$

(4) $\widetilde{\Delta}\left(\mathscr{F}_{\check{A}}+\mathscr{G}_{\check{B}}\right)=\widetilde{\Delta} \mathscr{F}_{\check{A}}+\widetilde{\Delta} \mathscr{G}_{\check{B}}$
The proof of the above proposition is easily obtained by using Definitions 11, 12, 16, and 19.

Definition 20. Let $\mathscr{F}_{\check{A}}$ be a GmPNSS over $\mathcal{U}$, then FalseFavorite operator on $\mathscr{F}_{\check{A}}$ can be represented by $\widetilde{\nabla} \mathscr{F}_{\breve{A}}$ and it is defined as follows:

$$
\widetilde{\nabla}_{\check{A}}=\left\{e,\left(u, s_{i} \bullet u_{A(e)}(u), 0, \min \left\{s_{i} \bullet w_{A(e)}(u)+s_{i} \bullet v_{A(e)}(u), 1\right\}: u \in U ; i \in 1,2,3, \ldots, m\right)\right\} .
$$

Proposition 7. Let $\mathscr{F}_{\check{A}}$ and $\mathscr{G}_{\check{B}}$ be GmPNSS over $\mathcal{U}$. Then,

(1) $\widetilde{\nabla} \widetilde{\nabla} \mathscr{F}_{\check{A}}=\widetilde{\nabla} \mathscr{F}_{\check{A}}$

(2) $\widetilde{\nabla}\left(\mathscr{F}_{\check{A}} \cup \mathscr{G}_{\check{B}}\right) \subseteq \widetilde{\nabla} \mathscr{F}_{\check{A}} \cup \widetilde{\nabla} \mathscr{G}_{\check{B}}$

(3) $\widetilde{\nabla}\left(\mathscr{F}_{\check{A}} \cap \mathscr{G}_{\tilde{B}}\right) \subseteq \widetilde{\nabla} \mathscr{F}_{\check{A}} \cap \widetilde{\nabla} \mathscr{G}_{\check{B}}$

(4) $\widetilde{\nabla}\left(\mathscr{F}_{\check{A}}+\mathscr{G}_{\breve{B}}\right)=\widetilde{\nabla} \mathscr{F}_{\check{A}}+\widetilde{\nabla} \mathscr{G}_{\check{B}}$

The proof of the above proposition is easily obtained by using Definitions 11, 12, 16, and 20.

Definition 21. Let $\mathscr{F}_{\check{A}}$ and $\mathscr{G}_{\check{B}}$ be GmPNSS; then their AND operator is represented by $\mathscr{F}_{\breve{A}} \wedge \mathscr{G}_{\check{B}}$ and it is defined as follows: $\mathscr{F}_{\check{A}} \wedge \mathscr{G}_{\check{B}}=\mathrm{l}_{A \times B}$, where $\mathrm{l}_{A \times B}(x, y)=\mathscr{F}_{A \check{(})}(x) \cap$ $\mathscr{G}_{B(e)}(y)$ for all $(x, y) \in A \times B$.

Definition 22. Let $\mathscr{F}_{\check{A}}$ and $\mathscr{G}_{\breve{B}}$ be GmPNSS; then their OR operator is represented by $\mathscr{F}_{\bar{A}} \vee \mathscr{G}_{\breve{B}}$ and it is defined as follows: $\mathscr{F}_{\breve{A}} \vee \mathscr{G}_{\breve{B}}=\mathrm{I}_{A \times B}$, where $\mathrm{I}_{A \times B}(x, y)=\mathscr{F}_{A(e)}(x) \cup$ $\mathscr{G}_{B(e)}(y)$ for all $(x, y) \in A \times B$.

Example 2. Reconsider Example 1.

$$
\begin{gathered}
\mathscr{F}_{\breve{A}(x)}=\left\{\begin{array}{l}
\left(x_{1},\left\{u_{1},(.5, .2, .1),(.3, .1, .2),(.6, .7, .8)\right)\left(u_{2},(.2, .3, .1),(.2, .1, .1),(.8, .6, .6)\right)\right. \\
\left(x_{2},\left\{u_{1},(.3, .1, .3),(0, .1, .3),(.5, .3, .5)\right)\left(u_{2},(.2, .2, .5),(.3, .1, .5),(.6, .5, .6)\right)\right.
\end{array}\right\}, \\
\mathscr{G}_{\breve{B}(x)}=\left\{\begin{array}{l}
\left(x_{2},\left\{u_{1},(.4, .3, .2),(.2, .3, .4),(.7, .4, .5)\right)\left(u_{2},(.1, .5, .1),(.3, .2, .2),(.5, .7, .4)\right)\right. \\
\left(x_{3},\left\{u_{1},(.2, .3, .2),(.1, .2, .2),(.4, .4, .5)\right)\left(u_{2},(.1, .1, .4),(.3, .3,1),(.5, .3, .1)\right)\right.
\end{array}\right\}, \\
\mathscr{F}_{\breve{A} \wedge} \wedge \mathscr{G}_{\check{B}}=\left\{\begin{array}{l}
\left(x_{1}, x_{2}\right),\left(u_{1},(.4, .3, .2),(.2, .3, .4),(.6, .7, .8)\right),\left(u_{2},(.1, .5, .1),(.2, .2, .2),(.5, .7, .6)\right), \\
\left(x_{1}, x_{3}\right),\left(u_{1},(.2, .3, .2),(.1, .2, .1),(.4, .7, .8)\right),\left(u_{2},(.1, .3, .4),(.2, .3, .1),(.5, .6, .6)\right), \\
\left(x_{2}, x_{2}\right),\left(u_{1},(.3, .1, .3),(.0, .1, .3),(.5, .3, .5)\right),\left(u_{2},(.2, .2, .5),(.3, .1, .5),(.6, .5, .6)\right), \\
\left(x_{2}, x_{3}\right),\left(u_{1},(.2, .1, .3),(.0, .2, .3),(.4, .4, .5)\right),\left(u_{2},(.1, .2, .5),(.3, .3, .5),(.5, .5, .6)\right)
\end{array}\right\} .
\end{gathered}
$$

Proposition 8. Let $\mathscr{F}_{\breve{A}}, \mathscr{G}_{\breve{B}}$, and $\mathscr{H}_{\check{C}}$ be GmPNSS. Then,

(1) $\mathscr{F}_{\check{A}} \vee \mathscr{G}_{\check{B}}=\mathscr{G}_{\check{B}} \vee \mathscr{F}_{\check{A}}$

(2) $\mathscr{F}_{\bar{A}} \wedge \mathscr{G}_{\check{B}}=\mathscr{G}_{\check{B}} \wedge \mathscr{F}_{\check{A}}$

(3) $\mathscr{F}_{\check{A}} \vee\left(\mathscr{G}_{\check{B}} \vee \mathscr{H}_{\check{C}}\right)=\left(\mathscr{F}_{\check{A}} \vee \mathscr{G}_{\check{B}}\right) \vee \mathscr{H}_{\check{C}}$

(4) $\mathscr{F}_{\grave{A}} \wedge\left(\mathscr{G}_{\breve{B}} \wedge \mathscr{H}_{\check{C}}\right)=\left(\mathscr{F}_{\bar{A}} \wedge \mathscr{G}_{\check{B}}\right) \wedge \mathscr{H}_{\check{C}}$

(5) $\left(\mathscr{F}_{\check{A}} \vee \mathscr{G}_{\check{B}}\right)^{c}=\mathscr{F}^{c}(\check{A}) \wedge \mathscr{G}^{c}(\check{B})$

(6) $\left(\mathscr{F}_{\check{A}} \wedge \mathscr{G}_{\check{B}}\right)^{c}=\mathscr{F}^{c}(\check{A}) \vee \mathscr{G}^{c}(\check{B})$
Proof. We can prove this easily by using Definitions 10, 21, and 22 .

Definition 23. Let $\mathscr{F}_{\check{A}}$ be a GmPNSS; then necessity operation on GmPNSS is represented by $\oplus \mathscr{F}_{\check{A}}$ and defined as follows:

$$
\oplus_{\check{A}}=\left\{\left(e,\left(u,\left(s_{i} \bullet u_{\check{A}(e)}(u), s_{i} \bullet v_{\check{A}(e)}(u), 1-s_{i} \bullet u_{\check{A}(e)}(u)\right)\right)\right): u \in U, e \in E ; i \in 1,2,3, \ldots, m\right\} .
$$


Definition 24. Let $\mathscr{F}_{\check{A}}$ be a GmPNSS; then possibility operation on GmPNSS is represented by $\otimes \mathscr{F}_{\check{A}}$ and defined as follows:

$$
\otimes \mathscr{F}_{\check{A}}=\left\{\left(e,\left(u,\left(1-s_{i} \bullet w_{\check{A}}(u), s_{i} \bullet v_{\check{A}(e)}(u), s_{i} \bullet w_{\check{A}}(u)\right)\right)\right): u \in U, e \in E ; i \in 1,2,3, \ldots, m\right\} .
$$

Proposition 9. Let $\mathscr{F}_{\check{A}}$ and $\mathscr{G}_{\breve{B}}$ be two GmPNSS. Then,

Proof. We know that

(1) $\oplus\left(\mathscr{F}_{\check{A}} \cup_{\varepsilon} \mathscr{G}_{\check{B}}\right)=\oplus \mathscr{G}_{\check{B}} \cup_{\varepsilon} \oplus \mathscr{F}_{\check{A}}$

(2) $\oplus\left(\mathscr{F}_{\check{A}} \cap_{\varepsilon} \mathscr{G}_{\check{B}}\right)=\oplus \mathscr{G}_{\check{B}} \cap_{\varepsilon} \oplus \mathscr{F}_{\check{A}}$

$$
\begin{aligned}
& \mathscr{F}_{\check{A}}=\left\{\left(e,\left(u,\left(s_{i} \bullet u_{\check{A}(e)}(u), s_{i} \bullet v_{\check{A}(e)}(u), s_{i} \bullet w_{\check{A}(e)}(u)\right)\right)\right): u \in U, e \in E ; i \in 1,2,3, \ldots, m\right\}, \\
& \mathscr{G}_{\check{B}}=\left\{\left(e,\left(u,\left(s_{i} \bullet u_{\check{B}(e)}(u), s_{i} \bullet v_{\check{B}(e)}(u), s_{i} \bullet w_{\breve{B}(e)}(u)\right)\right)\right): u \in U, e \in E ; i \in 1,2,3, \ldots, m\right\},
\end{aligned}
$$

$$
\begin{aligned}
& \text { are two GmPNSS. } \\
& \text { Let } \mathscr{F}_{\check{A}} \cup_{\varepsilon} \mathscr{G}_{\check{B}}=\mathscr{H}_{\check{C}} . \\
& u\left(\mathscr{H}_{\check{C}}\right)= \begin{cases}s_{i} \bullet u_{A(e)}(u), & \text { if } e \in A-B, \\
s_{i} \bullet u_{B(e)}(u), & \text { if } e \in B-A, \\
\max \left\{s_{i} \bullet u_{A(e)}(u), s_{i} \bullet u_{B(e)}(u)\right\}, & \text { if } e \in A \cap B,\end{cases} \\
& v\left(\mathscr{H}_{\check{C}}\right)= \begin{cases}s_{i} \bullet v_{A(e)}(u), & \text { if } e \in A-B, \\
s_{i} \bullet v_{B(e)}(u), & \text { if } e \in B-A, \\
\min \left\{s_{i} \bullet v_{A(e)}(u), s_{i} \bullet v_{B(e)}(u)\right\}, & \text { if } e \in A \cap B,\end{cases} \\
& w\left(\mathscr{H}_{\check{C}}\right)= \begin{cases}s_{i} \bullet w_{A(e)}(u), \\
s_{i} \bullet w_{B(e)}(u), \\
\min \left\{s_{i} \bullet w_{A(e)}(u), s_{i} \bullet w_{B(e)}(u)\right\}, & \text { if } e \in A \cap B .\end{cases}
\end{aligned}
$$

By using Definition 23,

$$
\oplus u\left(\mathscr{H}_{\check{C}}\right)= \begin{cases}s_{i} \bullet u_{A(e)}(u), & \text { if } e \in A-B, \\ s_{i} \bullet u_{B(e)}(u), & \text { if } e \in B-A, \\ \max \left\{s_{i} \bullet u_{A(e)}(u), s_{i} \bullet u_{B(e)}(u)\right\}, & \text { if } e \in A \cap B,\end{cases}
$$

$$
\oplus v\left(\mathscr{H}_{\check{C}}\right)= \begin{cases}s_{i} \bullet v_{A(e)}(u), & \text { if } e \in A-B, \\ s_{i} \bullet v_{B(e)}(u), & \text { if } e \in B-A, \\ \min \left\{s_{i} \bullet v_{A(e)}(u), s_{i} \bullet v_{B(e)}(u)\right\}, & \text { if } e \in A \cap B,\end{cases}
$$

$$
\oplus w\left(\mathscr{H}_{\check{C}}\right)= \begin{cases}s_{i} \bullet w_{A(e)}(u), & \text { if } e \in A-B, \\ s_{i} \bullet w_{B(e)}(u), & \text { if } e \in B-A, \\ \min \left\{s_{i} \bullet w_{A(e)}(u), s_{i} \bullet w_{B(e)}(u)\right\}, & \text { if } e \in A \cap B .\end{cases}
$$

Assume that $\oplus \mathscr{G}_{\breve{B}} \cup_{\varepsilon} \oplus \mathscr{F}_{\check{A}}=\aleph$, where $\oplus \mathscr{F}_{\check{A}}$ and $\oplus \mathscr{G}_{\breve{B}}$ are given as follows by using the definition of necessity operation:

$$
\begin{aligned}
& \oplus \mathscr{F}_{\check{A}}=\left\{\left(e,\left(u,\left(s_{i}^{\bullet} u_{\check{A}(e)}(u), s_{i} \bullet v_{\check{A}(e)}(u), 1-s_{i} \bullet u_{\check{A}(e)}(u)\right)\right)\right): u \in U, e \in E ; i \in 1,2,3, \ldots, m\right\}, \\
& \oplus \mathscr{G}_{\check{B}}=\left\{\left(e,\left(u,\left(s_{i} \bullet u_{\check{B}(e)}(u), s_{i} \bullet v_{\check{B}(e)}(u), 1-s_{i} \bullet u_{\check{B}(e)}(u)\right)\right)\right): u \in U, e \in E ; i \in 1,2,3, \ldots, m\right\} .
\end{aligned}
$$


Then, by using Definition 13,

$$
u(\aleph)= \begin{cases}s_{i} \bullet u_{A(e)}(u), & \text { if } e \in A-B, \\ s_{i} \bullet u_{B(e)}(u), & \text { if } e \in B-A, \\ \max \left\{s_{i} \bullet u_{A(e)}(u), s_{i} \bullet u_{B(e)}(u)\right\}, & \text { if } e \in A \cap B,\end{cases}
$$

$$
v(\aleph)= \begin{cases}s_{i} \bullet v_{A(e)}(u), & \text { if } e \in A-B, \\ s_{i} \bullet v_{B(e)}(u), & \text { if } e \in B-A, \\ \min \left\{s_{i} \bullet v_{A(e)}(u), s_{i} \bullet v_{B(e)}(u)\right\}, & \text { if } e \in A \cap B\end{cases}
$$

$$
w(\aleph)= \begin{cases}s_{i} \bullet u_{A(e)}(u), & \text { if } e \in A-B, \\ s_{i} \bullet u_{B(e)}(u), & \text { if } e \in B-A, \\ 1-\min \left\{s_{i} \bullet u_{A(e)}(u), s_{i} \bullet u_{B(e)}(u)\right\}, & \text { if } e \in A \cap B\end{cases}
$$

Consequently, $\oplus\left(\mathscr{H}_{\check{C}}\right)$ and $\aleph$ are the same, so

$$
\oplus\left(\mathscr{F}_{\check{A}} \cup_{\varepsilon} \mathscr{G}_{\check{B}}\right)=\oplus \mathscr{G}_{\check{B}} \cup_{\varepsilon} \oplus \mathscr{F}_{\check{A}} .
$$

Similarly, we can prove 2 .

Proposition 10. Let $\mathscr{F}_{\check{A}}$ and $\mathscr{G}_{\check{B}}$ be two GmPNSS. Then,

$(1) \otimes,\left(\mathscr{F}_{\check{A}} \cup_{\varepsilon} \mathscr{G}_{\check{B}}\right)=\otimes, \mathscr{G}_{\check{B}} \cup_{\varepsilon} \otimes, \mathscr{F}_{\check{A}}$

(2) $\otimes,\left(\mathscr{F}_{\check{A}} \cap_{\varepsilon} \mathscr{G}_{\check{B}}\right)=\otimes, \mathscr{G}_{\check{B}} \cap_{\varepsilon} \otimes, \mathscr{F}_{\check{A}}$

Proof. The proof is similar to that of Proposition 9.

Proposition 11. Let $\mathscr{F}_{\check{A}}$ and $\mathscr{G}_{\check{B}}$ be GmPNSS, then we have the following:
(1) $\oplus\left(\mathscr{F}_{\check{A}} \wedge \mathscr{G}_{\breve{B}}\right)=\oplus \mathscr{F}_{\check{A}} \wedge \oplus \mathscr{G}_{\check{B}}$
(2) $\oplus\left(\mathscr{F}_{\check{A}} \vee \mathscr{G}_{\breve{B}}\right)=\oplus \mathscr{F}_{\check{A}} \vee \oplus \mathscr{G}_{\check{B}}$
(3) $\otimes\left(\mathscr{F}_{\check{A}} \wedge \mathscr{G}_{\check{B}}\right)=\otimes \mathscr{F}_{\check{A}} \wedge \otimes \mathscr{G}_{\check{B}}$
$(4) \otimes\left(\mathscr{F}_{\check{A}} \vee \mathscr{G}_{\check{B}}\right)=\otimes \mathscr{F}_{\check{A}} \vee \otimes \mathscr{G}_{\check{B}}$

Proof. We know that $\mathscr{F}_{\check{A}}$ and $\mathscr{G}_{\check{B}}$ are GmPNSS:

$$
\begin{aligned}
& \mathscr{F}_{\check{A}}=\left\{\left(e,\left(u,\left(s_{i} \bullet u_{\check{A}(e)}(u), s_{i} \bullet v_{\check{A}(e)}(u), s_{i} \bullet w_{\check{A}(e)}(u)\right)\right)\right): u \in U, e \in E ; i \in 1,2,3, \ldots, m\right\}, \\
& \mathscr{G}_{\check{B}}=\left\{\left(e,\left(u,\left(s_{i} \bullet u_{\check{B}(e)}(u), s_{i} \bullet v_{\check{B}(e)}(u), s_{i} \bullet w_{\check{B}(e)}(u)\right)\right)\right): u \in U, e \in E ; i \in 1,2,3, \ldots, m\right\} .
\end{aligned}
$$

Let $\left(\mathscr{F}_{\check{A}} \wedge \mathscr{G}_{\breve{B}}\right)=\mathscr{H}_{\check{C}}$, where $\check{C}=\check{A} \times \check{B}$.

$$
\mathscr{H}_{\check{C}}=\left\{\left(e_{i}, e_{j}\right),\left[u, \min \left\{\begin{array}{c}
s_{i} \bullet u_{\check{A}(e)}(u) \\
s_{i} \bullet u_{\check{B}(e)}(u)
\end{array}\right\}, \max \left\{\begin{array}{c}
s_{i} \bullet v_{\check{A}(e)}(u) \\
s_{i} \bullet v_{\check{B}(e)}(u)
\end{array}\right\}, \max \left\{\begin{array}{c}
s_{i} \bullet w_{\check{A}(e)}(u) \\
s_{i} \bullet w_{\check{B}(e)}(u)
\end{array}\right\}\right]\right\} .
$$

By using Definition 23,

$$
\oplus \mathscr{H}_{\check{C}}=\left\{\left(e_{i}, e_{j}\right),\left[u, \min \left\{\begin{array}{c}
s_{i} \bullet \boldsymbol{u}_{\check{A}(e)}(u) \\
s_{i} \bullet u_{\check{B}(e)}(u)
\end{array}\right\}, \max \left\{\begin{array}{c}
s_{i} \bullet v_{\check{A}(e)}(u) \\
s_{i} \bullet v_{\check{B}(e)}(u)
\end{array}\right\}, 1-\min \left\{\begin{array}{c}
s_{i} \bullet u_{\check{A}(e)}(u) \\
s_{i} \bullet u_{\check{B}(e)}(u)
\end{array}\right\}\right]\right\} .
$$

We have

$$
\begin{aligned}
& \oplus \mathscr{F}_{\check{A}}=\left\{\left(e,\left(u,\left(s_{i} \bullet u_{\check{A}(e)}(u), s_{i} \bullet v_{\check{A}(e)}(u), 1-s_{i} \bullet u_{\check{A}(e)}(u)\right)\right)\right): u \in U, e \in E ; i \in 1,2,3, \ldots, m\right\}, \\
& \oplus \mathscr{G}_{\check{B}}=\left\{\left(e,\left(u,\left(s_{i} \bullet u_{\check{B}(e)}(u), s_{i} \bullet v_{\check{B}(e)}(u), 1-s_{i} \bullet u_{\check{B}(e)}(u)\right)\right)\right): u \in U, e \in E ; i \in 1,2,3, \ldots, m\right\} .
\end{aligned}
$$


By using Definition 21, we get

$$
\begin{aligned}
& \oplus \mathscr{F}_{\check{A}} \wedge \oplus \mathscr{G}_{\check{B}}=\left\{\left(e_{i}, e_{j}\right),\left[u, \min \left\{s_{i} \bullet u_{\check{A}(e)}(u), s_{i} \bullet u_{\check{B}(e)}(u)\right\}, \max \left\{s_{i} \bullet v_{\check{A}(e)}(u), s_{i} \bullet v_{\check{B}(e)}(u)\right\},\right.\right. \\
&\left.\left.\max \left\{\left(1-s_{i} \bullet u_{\check{A}(e)}(u)\right),\left(1-s_{i} \bullet u_{\check{B}(e)}(u)\right)\right\}\right]\right\}, \\
& \oplus \mathscr{F}_{\check{A}} \wedge \oplus \mathscr{G}_{\check{B}}=\left\{\left(e_{i}, e_{j}\right),\left[u, \min \left\{s_{i} \bullet u_{\check{A}(e)}(u), s_{i} \bullet u_{\check{B}(e)}(u)\right\}, \max \left\{s_{i} \bullet v_{\check{A}(e)}(u), s_{i} \bullet v_{\check{B}(e)}(u)\right\}, 1-\min \left\{s_{i} \bullet u_{\check{A}(e)}(u), s_{i} \bullet u_{\check{B}(e)}(u)\right\}\right]\right\} .
\end{aligned}
$$

So $\oplus\left(\mathscr{F}_{\check{A}} \wedge \mathscr{G}_{\breve{B}}\right)=\oplus \mathscr{F}_{\check{A}} \wedge \oplus \mathscr{G}_{\check{B}}$.

Similar to Assertion 1, we can prove 2, 3, and 4 .

\section{Distance and Similarity Measure of \\ Generalized Multipolar Neutrosophic}

Soft Set

In this section, we introduce the Hamming distance and Euclidean distance between two GmPNSS and develop the similarity measure by using these distances.
Definition 25. $\mathcal{U}$ and $E$ are a universal set and a set of attributes, respectively; assume that $\operatorname{GmPNSS}(\mathcal{U})$ represents the collection of all GmPNSS. Consider $\left(\Phi_{\mathscr{F}}, E\right)$ as well as $\left(\varphi_{\mathscr{G}}, E\right) \in \mathrm{GmPNSS}$ and there exists a mapping $\Phi_{\mathscr{F}}, \varphi_{\mathscr{G}}: E$ $\longrightarrow$ GmPNSS$(\mathcal{U})$; after that, we tend to establish the distances between $\left(\Phi_{\mathscr{F}}, E\right)$ and $\left(\varphi_{\mathscr{G}}, E\right)$ as follows.

\subsection{Hamming Distance}

$$
\begin{aligned}
d_{\mathrm{GmPNSS}}^{H}\left(\Phi_{\mathscr{F}}(e), \varphi_{\mathscr{G}}(e)\right)= & \frac{1}{2 m}\left\{\sum_{i=1}^{m} \sum_{j=1}^{p}\left(\left|s_{i} \bullet u_{\Phi_{\mathscr{F}}}\left(u_{j}\right)-s_{i} \bullet u_{\varphi_{\mathscr{G}}}\left(u_{j}\right)\right|\right)+\left(\left|s_{i} \bullet v_{\Phi_{\mathscr{F}}}\left(u_{j}\right)-s_{i} \bullet v_{\varphi_{\mathscr{G}}}\left(u_{j}\right)\right|\right)\right. \\
& \left.+\left(\left|s_{i} \bullet w_{\Phi_{\mathscr{F}}}\left(u_{j}\right)-s_{i} \bullet w_{\varphi_{\mathscr{G}}}\left(u_{j}\right)\right|\right)\right\} .
\end{aligned}
$$

\subsection{Normalized Hamming Distance}

$$
\begin{aligned}
d_{\mathrm{GmPNSS}}^{\mathrm{NH}}\left(\Phi_{\mathscr{F}}(e), \varphi_{\mathscr{G}}(e)\right)= & \frac{1}{2 m p}\left\{\sum_{i=1}^{m} \sum_{j=1}^{p}\left(\left|s_{i} \bullet u_{\Phi_{\mathscr{F}}}\left(u_{j}\right)-s_{i} \bullet u_{\varphi_{\mathscr{G}}}\left(u_{j}\right)\right|\right)+\left(\left|s_{i} \bullet v_{\Phi_{\mathscr{F}}}\left(u_{j}\right)-s_{i} \bullet v_{\varphi_{\mathscr{G}}}\left(u_{j}\right)\right|\right)\right. \\
& \left.+\left(\left|s_{i} \bullet w_{\Phi_{\mathscr{F}}}\left(u_{j}\right)-s_{i} \bullet w_{\varphi_{\mathscr{G}}}\left(u_{j}\right)\right|\right)\right\} .
\end{aligned}
$$

\subsection{Euclidean Distance}

$$
\begin{aligned}
d_{\mathrm{GmPNSS}}^{E}\left(\Phi_{\mathscr{F}}(e), \varphi_{\mathscr{G}}(e)\right)= & \left(\frac { 1 } { 2 m } \left\{\sum_{i=1}^{m} \sum_{j=1}^{p}\left(\left|s_{i} \bullet u_{\Phi_{\mathscr{F}}}\left(u_{j}\right)-s_{i} \bullet u_{\varphi_{\mathscr{G}}}\left(u_{j}\right)\right|\right)^{2}+\left(\left|s_{i} \bullet v_{\Phi_{\mathscr{F}}}\left(u_{j}\right)-s_{i} \bullet v_{\varphi_{\mathscr{G}}}\left(u_{j}\right)\right|\right)^{2}\right.\right. \\
& \left.\left.+\left(\left|s_{i} \bullet w_{\Phi_{\mathscr{F}}}\left(u_{j}\right)-s_{i} \bullet w_{\varphi_{\mathscr{G}}}\left(u_{j}\right)\right|\right)^{2}\right\}\right)^{1 / 2} \cdot
\end{aligned}
$$




$$
\begin{aligned}
d_{\mathrm{GmPNSS}}^{\mathrm{NE}}\left(\Phi_{\mathscr{F}}(e), \varphi_{\mathscr{G}}(e)\right)= & \left(\frac { 1 } { 2 m p } \left\{\sum_{i=1}^{m} \sum_{j=1}^{p}\left(\left|s_{i} \bullet u_{\Phi_{\mathscr{F}}}\left(u_{j}\right)-s_{i} \bullet u_{\varphi_{\mathscr{G}}}\left(u_{j}\right)\right|\right)^{2}+\left(\left|s_{i} \bullet v_{\Phi_{\mathscr{F}}}\left(u_{j}\right)-s_{i} \bullet v_{\varphi_{\mathscr{G}}}\left(u_{j}\right)\right|\right)^{2}\right.\right. \\
& \left.\left.+\left(\left|s_{i} \bullet w_{\Phi_{\mathscr{F}}}\left(u_{j}\right)-s_{i} \bullet w_{\varphi_{\mathscr{G}}}\left(u_{j}\right)\right|\right)^{2}\right\}\right)^{1 / 2}
\end{aligned}
$$

\subsection{Weighted Distance}

$$
\begin{aligned}
d_{\mathrm{GmPNSS}}^{w}\left(\Phi_{\mathscr{F}}(e), \varphi_{\mathscr{G}}(e)\right)= & \left(\frac { 1 } { 2 m } \left\{\sum _ { i = 1 } ^ { m } \sum _ { j = 1 } ^ { p } w _ { i } \left\{\left(\left|s_{i} \bullet u_{\Phi_{\mathscr{F}}}\left(u_{j}\right)-s_{i} \bullet u_{\varphi_{\mathscr{G}}}\left(u_{j}\right)\right|\right)^{r}+\left(\left|s_{i} \bullet v_{\Phi_{\mathscr{F}}}\left(u_{j}\right)-s_{i} \bullet v_{\varphi_{\mathscr{G}}}\left(u_{j}\right)\right|\right)^{r}\right.\right.\right. \\
& \left.\left.\left.+\left(\left|s_{i} \bullet w_{\Phi_{\mathscr{F}}}\left(u_{j}\right)-s_{i} \bullet w_{\varphi_{\mathscr{G}}}\left(u_{j}\right)\right|\right)^{r}\right\}\right\}\right)^{1 / r} \cdot
\end{aligned}
$$

where $r>0$ and $w=\left(w_{1}, w_{2}, w_{3}, \ldots, w_{n}\right)^{T}$ is a weight vector of $e_{i}(i=1,2,3, \ldots, n)$. If $r=1$ and $r=2$, then equation (5) becomes the weighted hamming and weighted Euclidean distances, respectively.

Definition 26. $\mathcal{U}$ and $E$ are a universal set and a set of attributes, respectively, and $\left(\Phi_{\mathscr{F}}, E\right)$ and $\left(\varphi_{\mathscr{G}}, E\right)$ are two GmPNSS $(\mathcal{U})$. Then similarity measure based on Definition 25 between $\left(\Phi_{\mathscr{F}}, E\right)$ and $\left(\varphi_{\mathscr{G}}, E\right)$ is defined as follows:

$$
S_{\mathrm{GmPNSS}}\left(\Phi_{\mathscr{F}}, \varphi_{\mathscr{G}}\right)=\frac{1}{1+d\left(\Phi_{\mathscr{F}}, \varphi_{\mathscr{G}}\right)} .
$$

Another similarity measure between $\left(\Phi_{\mathscr{F}}, E\right)$ and $\left(\varphi_{\mathscr{G}}, E\right)$ is defined as

$$
S_{\mathrm{GmPNSS}}\left(\Phi_{\mathscr{F}}, \varphi_{\mathscr{G}}\right)=e^{-\beta d\left(\Phi_{\mathscr{F}}, \varphi_{\mathscr{G}}\right)},
$$

where $\beta$ is a steepness measure and a positive real number.

Definition 27. $\mathcal{U}$ and $E$ are a universal set and a set of attributes, respectively, and $\left(\Phi_{\mathscr{F}}, E\right)$ and $\left(\varphi_{\mathscr{G}}, E\right)$ are two $\operatorname{GmPNSS}(\mathcal{U})$. Then, the distances between $\left(\Phi_{\mathscr{F}}, E\right)$ and $\left(\varphi_{\mathscr{G}}\right.$, E) are defined as follows:

$$
\begin{aligned}
d_{\mathrm{GmPNSS}}\left(\Phi_{\mathscr{F}}(e), \varphi_{\mathscr{G}}(e)\right)= & \frac{1}{2 m}\left\{\sum_{i=1}^{m} \sum_{j=1}^{p}\left(\left|s_{i} \bullet u_{\Phi_{\mathscr{F}}}\left(u_{j}\right)-s_{i} \bullet u_{\varphi_{\mathscr{G}}}\left(u_{j}\right)\right|\right)^{r}+\left(\left|s_{i} \bullet v_{\Phi_{\mathscr{F}}}\left(u_{j}\right)-s_{i} \bullet v_{\varphi_{\mathscr{G}}}\left(u_{j}\right)\right|\right)^{r}\right. \\
& \left.\left.+\left(\left|s_{i} \bullet w_{\Phi_{\mathscr{F}}}\left(u_{j}\right)-s_{i} \bullet w_{\varphi_{\mathscr{G}}}\left(u_{j}\right)\right|\right)^{r}\right\}\right)^{1 / r}, \\
d_{\mathrm{GmPNSS}}\left(\Phi_{\mathscr{F}}(e), \varphi_{\mathscr{G}}(e)\right)= & \left(\frac { 1 } { 2 m p } \left\{\sum_{i=1}^{m} \sum_{j=1}^{p}\left(\left|s_{i} \bullet u_{\Phi_{\mathscr{F}}}\left(u_{j}\right)-s_{i} \bullet u_{\varphi_{\mathscr{G}}}\left(u_{j}\right)\right|\right)^{r}+\left(\left|s_{i} \bullet v_{\Phi_{\mathscr{F}}}\left(u_{j}\right)-s_{i} \bullet v_{\varphi_{\mathscr{G}}}\left(u_{j}\right)\right|\right)^{r}\right.\right. \\
& \left.\left.+\left(\left|s_{i} \bullet w_{\Phi_{\mathscr{F}}}\left(u_{j}\right)-s_{i} \bullet w_{\varphi_{\mathscr{G}}}\left(u_{j}\right)\right|\right)^{r}\right\}\right)^{1 / r} .
\end{aligned}
$$

where $r>0$, and equations (8) and (9) are reduced to equations (1) and (2), respectively, if $r=1$. Similarly, if $r=2$, then equations (8) and (9) are reduced to equations (3) and (4), respectively. 
Definition 28. Similarity measure between two GmPNSS $\left(\Phi_{\mathscr{F}}, E\right)$ and $\left(\varphi_{\mathscr{G}}, E\right)$ based on the weighted distance of $\left(\Phi_{\mathscr{F}}\right.$, $E)$ and $\left(\varphi_{\mathscr{G}}, E\right)$ is defined as follows:

$$
S_{\mathrm{GmPNSS}}\left(\Phi_{\mathscr{F}}, \varphi_{\mathscr{G}}\right)=\frac{1}{1+d_{\mathrm{GmPNSS}}^{w}\left(\Phi_{\mathscr{F}}, \varphi_{\mathscr{G}}\right)}
$$

Definition 29. Let $\Phi_{\mathscr{F}}$ and $\varphi_{\mathscr{G}}$ be GmPNSS over the universal set; then $\Phi_{\mathscr{F}}$ and $\varphi_{\mathscr{G}}$ are said to be $\alpha$-similar if and only if $S_{\mathrm{GmPNSS}}\left(\Phi_{\mathscr{F}}, \varphi_{\mathscr{G}}\right) \geq \alpha$ for $\alpha \in(0,1)$. If $S_{\mathrm{GmPNSS}}\left(\Phi_{\mathscr{F}}, \varphi_{\mathscr{G}}\right)>$ $1 / 2$, then we can say that $\Phi_{\mathscr{F}}$ and $\varphi_{\mathscr{G}}$ are significantly similar.

\section{Correlation Coefficient of Generalized Multipolar Neutrosophic Soft Set}

In this section, we propose the concept of correlation coefficient and weighted correlation coefficient of GmPNSS with some properties.

Definition 30. Let

$$
\begin{aligned}
\mathscr{F}_{\check{A}} & =\left\{u_{k},\left(s_{i} \bullet u_{\breve{A}}\left(u_{k}\right), s_{i} \bullet v_{\check{A}}\left(u_{k}\right), s_{i} \bullet w_{\check{A}}\left(u_{k}\right)\right): u_{k} \in U ; i \in 1,2,3, \ldots, m\right\}, \\
G_{\breve{B}} & =\left\{u_{k},\left(s_{i} \bullet u_{\breve{B}}\left(u_{k}\right), s_{i} \bullet v_{\breve{B}}\left(u_{k}\right), s_{i} \bullet w_{\breve{B}}\left(u_{k}\right)\right): u_{k} \in U ; i \in 1,2,3, \ldots, m\right\},
\end{aligned}
$$

be two GmPNSS over a set of parameters $E=\left\{x_{1}, x_{2}, x_{3}, \ldots, x_{n}\right\}$.
Then, informational neutrosophic energies of two GmPNSS can be expressed as follows:

$$
\begin{aligned}
& \varepsilon_{\mathrm{GmPNSS}}\left(F_{\breve{A}}\right)=\sum_{j=1}^{z} \sum_{k=1}^{t}\left(\left(s_{i} \bullet u_{\breve{A}_{j}}\left(u_{k}\right)\right)^{2}+\left(s_{i} \bullet v_{\breve{A}_{j}}\left(u_{k}\right)\right)^{2}+\left(s_{i} \bullet w_{\breve{A}_{j}}\left(u_{k}\right)\right)^{2}\right), \\
& \varepsilon_{\mathrm{GmPNSS}}\left(G_{\breve{B}}\right)=\sum_{j=1}^{z} \sum_{k=1}^{t}\left(\left(s_{i} \bullet u_{\bar{B}_{j}}\left(u_{k}\right)\right)^{2}+\left(s_{i} \bullet v_{\bar{B}_{j}}\left(u_{k}\right)\right)^{2}+\left(s_{i} \bullet w_{\breve{B}_{j}}\left(u_{k}\right)\right)^{2}\right) .
\end{aligned}
$$

Definition 31. The correlation of two GmPNSS can be presented as follows:

$$
\zeta_{\mathrm{GmPNSS}}\left(F_{\breve{A}}, G_{\breve{B}}\right)=\sum_{j=1}^{z} \sum_{k=1}^{t}\left\{\left(s_{i} \bullet u_{\breve{A}_{j}}\left(u_{k}\right) s_{i} \bullet u_{\breve{B}_{j}}\left(u_{k}\right)+s_{i} \bullet v_{\breve{A}_{j}}\left(u_{k}\right) s_{i} \bullet v_{\breve{B}_{j}}\left(u_{k}\right)+s_{i} \bullet w_{\breve{A}_{j}}\left(u_{k}\right) s_{i} \bullet w_{\breve{B_{j}}}\left(u_{k}\right)\right): i \in 1,2,3, \ldots, m\right\} .
$$

Definition 32. Let $F_{\breve{A}}$ and $G_{\breve{B}}$ be two GmPNSS; then the CC between them can be defined as follows:

$$
\begin{aligned}
& R_{\mathrm{GmPNSS}}\left(F_{\breve{A}}, G_{\breve{B}}\right)=\frac{\zeta_{\mathrm{GmPNSS}}\left(F_{\breve{A}}, G_{\breve{B}}\right)}{\sqrt{\varepsilon_{\mathrm{GmPNSS}}\left(F_{\breve{A}}, F_{\breve{A}}\right) \cdot \varepsilon_{\mathrm{GmPNSS}}\left(G_{\breve{B}}, G_{\breve{B}}\right)}}, \\
& R_{\mathrm{GmPNSS}}\left(F_{\breve{A}}, G_{\breve{B}}\right)=\frac{\sum_{j=1}^{z} \sum_{k=1}^{t}\left(s_{i} \bullet u_{\breve{A}_{j}}\left(u_{k}\right) s_{i} \bullet u_{\breve{B}_{j}}\left(u_{k}\right)+s_{i} \bullet v_{\bar{A}_{j}}\left(u_{k}\right) s_{i} \bullet v_{\breve{B}_{j}}\left(u_{k}\right)+s_{i} \bullet w_{\breve{A}_{j}}\left(u_{k}\right) s_{i} \bullet w_{\breve{B}_{j}}\left(u_{k}\right)\right)}{\sqrt{\sum_{j=1}^{z} \sum_{k=1}^{t}\left(\left(s_{i} \bullet u_{\breve{A}_{j}}\left(u_{k}\right)\right)^{2}+\left(s_{i} \bullet v_{\breve{A}_{j}}\left(u_{k}\right)\right)^{2}+\left(s_{i} \bullet w_{\breve{A}_{j}}\left(u_{k}\right)\right)^{2}\right)} \sqrt{\sum_{j=1}^{z} \sum_{k=1}^{t}\left(\left(s_{i} \bullet u_{\breve{B}_{j}}\left(u_{k}\right)\right)^{2}+\left(s_{i} \bullet v_{\breve{B}_{j}}\left(u_{k}\right)\right)^{2}+\left(s_{i} \bullet w_{\breve{B}_{j}}\left(u_{k}\right)\right)^{2}\right)}} .
\end{aligned}
$$


Proposition 12. Let $F_{\breve{A}}$ and $G_{\breve{B}}$ be two GmPNSS; then the CC $R_{\text {GmPNSS }}\left(F_{\breve{A}}, G_{\breve{B}}\right)$ between them satisfies the following properties:

(1) $0 \leq R_{\text {GmPNSS }}\left(F_{\breve{A}}, G_{\breve{B}}\right) \leq 1$

(2) $R_{\text {GmPNSS }}\left(F_{\breve{A}}, G_{\breve{B}}\right)=R_{\text {GmPNSS }}\left(G_{\breve{B}}, F_{\breve{A}}\right)$

(3) If $F_{\breve{A}}=G_{\breve{B}}$, that is, $s_{i} \bullet u_{\breve{A}}\left(u_{k}\right)=s_{i} \bullet u_{\breve{B}_{i}}\left(u_{k}\right), s_{i} \bullet v_{\breve{A}}$ $\left(u_{k}\right)=s_{i} \bullet v_{\bar{B}_{j}}\left(u_{k}\right)$, and $s_{i} \bullet \vec{w}_{\bar{A}_{j}}\left(u_{k}\right)=s_{i} \bullet \vec{\omega}_{\breve{B}_{j}}\left(u_{k}\right)$ for all $j, k$, where $i \in 1,2,3, \ldots, m$, then $R_{G m P N S S}$ $\left(F_{\breve{A}}, G_{\breve{B}}\right)=1$

Proof. $R_{\text {GmPNSS }}\left(F_{\breve{A}}, G_{\breve{B}}\right) \geq 0$ is trivial, so we just need to prove that $R_{\text {GmPNSS }}\left(F_{\breve{A}}, G_{\breve{B}}\right) \leq 1$.

We know that

$$
\begin{aligned}
& \zeta_{\text {GmPNSS }}\left(F_{\breve{A}}, G_{\breve{B}}\right)=\sum_{j=1}^{z} \sum_{k=1}^{t}\left(s_{i} \cdot u_{\breve{A}_{j}}\left(u_{k}\right) s_{i} \cdot u_{\breve{B}_{j}}\left(u_{k}\right)+s_{i} \cdot v_{\breve{A}_{j}}\left(u_{k}\right) s_{i} \cdot v_{\breve{B}_{j}}\left(u_{k}\right)+s_{i} \cdot w_{\breve{A}_{j}}\left(u_{k}\right) s_{i} \cdot w_{\breve{B}_{j}}\left(u_{k}\right)\right) \\
& =\sum_{j=1}^{z}\left(s_{i} \cdot u_{\breve{A}_{j}}\left(u_{1}\right) s_{i} \cdot u_{\breve{B}_{j}}\left(u_{1}\right)+s_{i} \cdot v_{\breve{A}_{j}}\left(u_{1}\right) s_{i} \cdot v_{\bar{B}_{j}}\left(u_{1}\right)+s_{i} \cdot w_{\breve{A}_{j}}\left(u_{1}\right) s_{i} \cdot w_{\breve{B}_{j}}\left(u_{1}\right)\right) \\
& +\sum_{j=1}^{z}\left(s_{i} \cdot u_{\breve{A}_{j}}\left(u_{2}\right) s_{i} \cdot u_{\breve{B}_{j}}\left(u_{2}\right)+s_{i} \cdot v_{\breve{A}_{j}}\left(u_{2}\right) s_{i} \cdot v_{\breve{B}_{j}}\left(u_{2}\right)+s_{i} \cdot w_{\breve{A}_{j}}\left(u_{2}\right) s_{i} \cdot w_{\breve{B}_{j}}\left(u_{2}\right)\right)+ \\
& +\sum_{j=1}^{z}\left(s_{i} \cdot u_{\breve{A}_{j}}\left(u_{t}\right) s_{i} \cdot u_{\breve{B}_{j}}\left(u_{t}\right)+s_{i} \cdot v_{\breve{A}_{j}}\left(u_{t}\right) s_{i} \cdot v_{\breve{B}_{j}}\left(u_{t}\right)+s_{i} \cdot w_{\breve{A}_{j}}\left(u_{t}\right) s_{i} \cdot w_{\breve{B}_{j}}\left(u_{t}\right)\right) \\
& = \\
& \left\{\begin{array}{c}
\left(s_{i} \cdot u_{\breve{A}_{1}}\left(u_{1}\right) s_{i} \cdot u_{\breve{B}_{1}}\left(u_{1}\right)+s_{i} \cdot v_{\breve{A}_{1}}\left(u_{1}\right) s_{i} \cdot v_{\breve{B}_{1}}\left(u_{1}\right)+s_{i} \cdot w_{\breve{A}_{1}}\left(u_{1}\right) s_{i} \cdot w_{\breve{B}_{1}}\left(u_{1}\right)\right)+ \\
\left(s_{i} \cdot u_{\breve{A}_{2}}\left(u_{1}\right) s_{i} \cdot u_{\breve{B}_{2}}\left(u_{1}\right)+s_{i} \cdot v_{\breve{A}_{2}}\left(u_{1}\right) s_{i} \cdot v_{\breve{B}_{2}}\left(u_{1}\right)+s_{i} \cdot w_{\breve{A}_{2}}\left(u_{1}\right) s_{i} \cdot w_{\breve{B}_{2}}\left(u_{1}\right)\right)+\cdots+ \\
\left(s_{i} \cdot u_{\breve{A}_{z}}\left(u_{1}\right) s_{i} \cdot u_{\breve{B}_{z}}\left(u_{1}\right)+s_{i} \cdot v_{\breve{A}_{z}}\left(u_{1}\right) s_{i} \cdot v_{\breve{B}_{z}}\left(u_{1}\right)+s_{i} \cdot w_{\breve{A}_{z}}\left(u_{1}\right) s_{i} \cdot w_{\breve{B}_{z}}\left(u_{1}\right)\right)+
\end{array}\right\} \\
& + \\
& \left\{\begin{array}{c}
\left(s_{i} \cdot u_{\breve{A}_{1}}\left(u_{2}\right) s_{i} \cdot u_{\breve{B}_{1}}\left(u_{2}\right)+s_{i} \cdot v_{\breve{A}_{1}}\left(u_{2}\right) s_{i} \cdot v_{\breve{B}_{1}}\left(u_{2}\right)+s_{i} \cdot w_{\breve{A}_{1}}\left(u_{2}\right) s_{i} \cdot w_{\breve{B}_{1}}\left(u_{2}\right)\right)+ \\
\left(s_{i} \cdot u_{\breve{A}_{2}}\left(u_{2}\right) s_{i} \cdot u_{\breve{B}_{2}}\left(u_{2}\right)+s_{i} \cdot v_{\breve{A}_{2}}\left(u_{2}\right) s_{i} \cdot v_{\breve{B}_{2}}\left(u_{2}\right)+s_{i} \cdot w_{\breve{A}_{2}}\left(u_{2}\right) s_{i} \cdot w_{\breve{B}_{2}}\left(u_{2}\right)\right)+\cdots+ \\
\left(s_{i} \cdot u_{\breve{A}_{z}}\left(u_{2}\right) s_{i} \cdot u_{\breve{B}_{z}}\left(u_{2}\right)+s_{i} \cdot v_{\breve{A}_{z}}\left(u_{2}\right) s_{i} \cdot v_{\breve{B}_{z}}\left(u_{2}\right)+s_{i} \cdot w_{\breve{A}_{z}}\left(u_{2}\right) s_{i} \cdot w_{\breve{B}_{z}}\left(u_{2}\right)\right)+
\end{array}\right\} \\
& +\cdots+
\end{aligned}
$$

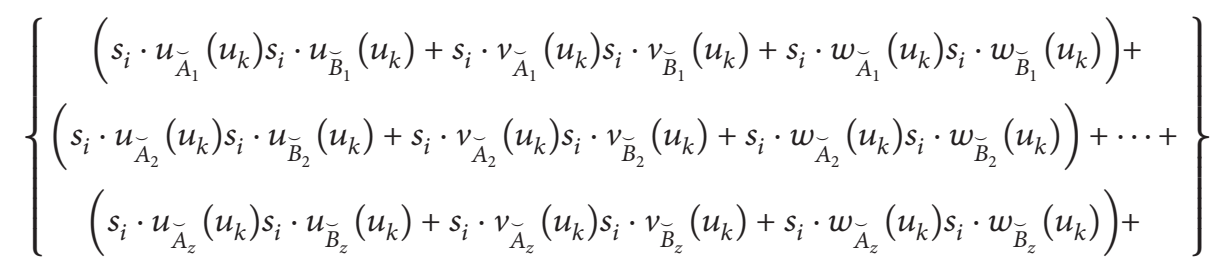

$$
\begin{aligned}
& =\sum_{j=1}^{z}\left(s_{i} \cdot u_{\breve{A}_{j}}\left(u_{1}\right) s_{i} \cdot u_{\breve{B}_{j}}\left(u_{1}\right)+s_{i} \cdot u_{\breve{A}_{j}}\left(u_{2}\right) s_{i} \cdot u_{\breve{B}_{j}}\left(u_{2}\right)+\cdots+s_{i} \cdot u_{\breve{A}_{j}}\left(u_{t}\right) s_{i} \cdot u_{\breve{B}_{j}}\left(u_{t}\right)\right) \\
& +\sum_{j=1}^{z}\left(s_{i} \cdot v_{\breve{A}_{j}}\left(u_{1}\right) s_{i} \cdot v_{\breve{B}_{j}}\left(u_{1}\right)+s_{i} \cdot v_{\bar{A}_{j}}\left(u_{2}\right) s_{i} \cdot v_{\bar{B}_{j}}\left(u_{2}\right)+\cdots+s_{i} \cdot v_{\breve{A}_{j}}\left(u_{t}\right) s_{i} \cdot v_{\bar{B}_{j}}\left(u_{t}\right)\right) \\
& +\sum_{j=1}^{z}\left(s_{i} \cdot w_{\breve{A}_{j}}\left(u_{1}\right) s_{i} \cdot w_{\breve{B}_{j}}\left(u_{1}\right)+s_{i} \cdot w_{\breve{A}_{j}}\left(u_{2}\right) s_{i} \cdot w_{\breve{B}_{j}}\left(u_{2}\right)+\cdots+s_{i} \cdot w_{\breve{A}_{j}}\left(u_{t}\right) s_{i} \cdot w_{\breve{B}_{j}}\left(u_{t}\right)\right) \text {. }
\end{aligned}
$$


By using Cauchy-Schwarz inequality, we get

$$
\begin{aligned}
& \left(\zeta_{\text {GmPNSS }}\left(F_{\breve{A}}, G_{\breve{B}}\right)\right)^{2} \leq \\
& \left\{\begin{array}{c}
\sum_{j=1}^{z}\left(\left(s_{i} \cdot u_{\breve{A}_{j}}\left(u_{1}\right)\right)^{2}+\left(s_{i} \cdot u_{\breve{A}_{j}}\left(u_{2}\right)\right)^{2}+\cdots+\left(s_{i} \cdot u_{\breve{A}_{j}}\left(u_{t}\right)\right)^{2}\right)+ \\
\sum_{j=1}^{z}\left(\left(s_{i} \cdot v_{\breve{A}_{j}}\left(u_{1}\right)\right)^{2}+\left(s_{i} \cdot v_{\breve{A}_{j}}\left(u_{2}\right)\right)^{2}+\cdots+\left(s_{i} \cdot v_{\breve{A}_{j}}\left(u_{t}\right)\right)^{2}\right)+\cdots+ \\
\sum_{j=1}^{z}\left(\left(s_{i} \cdot w_{\breve{A}_{j}}\left(u_{1}\right)\right)^{2}+\left(s_{i} \cdot w_{\breve{A}_{j}}\left(u_{2}\right)\right)^{2}+\cdots+\left(s_{i} \cdot w_{\breve{A}_{j}}\left(u_{t}\right)\right)^{2}\right)
\end{array}\right\} \\
& \times\left\{\begin{array}{c}
\sum_{j=1}^{z}\left(\left(s_{i} \cdot u_{\breve{B}_{j}}\left(u_{1}\right)\right)^{2}+\left(s_{i} \cdot u_{\breve{B}_{j}}\left(u_{2}\right)\right)^{2}+\cdots+\left(s_{i} \cdot u_{\breve{B}_{j}}\left(u_{t}\right)\right)^{2}\right)+ \\
\sum_{j=1}^{z}\left(\left(s_{i} \cdot v_{\breve{B}_{j}}\left(u_{1}\right)\right)^{2}+\left(s_{i} \cdot v_{\breve{B}_{j}}\left(u_{2}\right)\right)^{2}+\cdots+\left(s_{i} \cdot v_{\breve{B}_{j}}\left(u_{t}\right)\right)^{2}\right)+\cdots+ \\
\sum_{j=1}^{z}\left(\left(s_{i} \cdot w_{\breve{B}_{j}}\left(u_{1}\right)\right)^{2}+\left(s_{i} \cdot w_{\breve{B}_{j}}\left(u_{2}\right)\right)^{2}+\cdots+\left(s_{i} \cdot w_{\breve{B}_{j}}\left(u_{t}\right)\right)^{2}\right)
\end{array}\right\} \\
& = \\
& \left\{\sum_{j=1}^{z} \sum_{k=1}^{t}\left(\left(s_{i} \cdot u_{\breve{A}_{j}}\left(u_{t}\right)\right)^{2}+\left(s_{i} \cdot v_{\breve{A}_{j}}\left(u_{t}\right)\right)^{2}+\left(s_{i} \cdot w_{\breve{A}_{j}}\left(u_{t}\right)\right)^{2}\right)\right\} \\
& \times\left\{\sum_{j=1}^{z} \sum_{k=1}^{t}\left(\left(s_{i} \cdot u_{\breve{B}_{j}}\left(u_{t}\right)\right)^{2}+\left(s_{i} \cdot v_{\breve{B}_{j}}\left(u_{t}\right)\right)^{2}+\left(s_{i} \cdot w_{\breve{B}_{j}}\left(u_{t}\right)\right)^{2}\right)\right\} \\
& =\varepsilon_{\mathrm{GmPNSS}}\left(F_{\breve{A}}\right) \bullet \varepsilon_{\mathrm{GmPNSS}}\left(G_{\breve{B}}\right) \text {. }
\end{aligned}
$$

Therefore,

$\left(\zeta_{\text {GmPNSS }}\left(F_{\breve{A}}, G_{\breve{B}}\right)\right)^{2} \leq \varepsilon_{\text {GmPNSS }}\left(F_{\breve{A}}\right) \cdot \varepsilon_{\text {GmPNSS }}\left(G_{\breve{B}}\right)$. Hence, by using Definition 32 , we get $R_{\text {GmPNSS }}\left(F_{\breve{A}}, G_{\breve{B}}\right) \leq 1$, so $0 \leq R_{\mathrm{GmPNSS}}\left(F_{\breve{A}}, G_{\breve{B}}\right) \leq 1$.
Proof. The proof is obvious.

Proof. We know that

$$
R_{\mathrm{GmPNSS}}\left(F_{\breve{A}}, G_{\breve{B}}\right)=\frac{\sum_{j=1}^{z} \sum_{k=1}^{t}\left(s_{i} \cdot u_{\breve{B}_{j}}\left(u_{k}\right) s_{i} \cdot u_{\breve{B}_{j}}\left(u_{k}\right)+s_{i} \cdot v_{\breve{B}_{j}}\left(u_{k}\right) s_{i} \cdot v_{\bar{B}_{j}}\left(u_{k}\right)+s_{i} \cdot w_{\breve{B}_{j}}\left(u_{k}\right) s_{i} \cdot w_{\breve{B}_{j}}\left(u_{k}\right)\right)}{\sqrt{\sum_{j=1}^{z} \sum_{k=1}^{t}\left(\left(s_{i} \cdot u_{\breve{B}_{j}}\left(u_{k}\right)\right)^{2}+\left(s_{i} \cdot v_{\breve{B}_{j}}\left(u_{k}\right)\right)^{2}+\left(s_{i} \cdot w_{\breve{B}_{j}}\left(u_{k}\right)\right)^{2}\right)} \sqrt{\sum_{j=1}^{z} \sum_{k=1}^{t}\left(\left(s_{i} \cdot u_{\breve{B}_{j}}\left(u_{k}\right)\right)^{2}+\left(s_{i} \cdot v_{\breve{B}_{j}}\left(u_{k}\right)\right)^{2}+\left(s_{i} \cdot w_{\bar{B}_{j}}\left(u_{k}\right)\right)^{2}\right)}} .
$$

As we know that $s_{i} \cdot u_{\breve{A}_{\breve{j}}}\left(u_{k}\right)=s_{i} \cdot u_{\breve{B}_{j}}\left(u_{k}\right)$, $s_{i} \cdot v_{\breve{A^{\prime}}}\left(u_{k}\right)=s_{i} \cdot v_{\breve{B}_{j}}\left(u_{k}\right)$, and $\left.s_{i} \cdot w_{\breve{A}_{h}}\left(u_{k}\right)_{k}\right)=s_{i} \cdot w_{\breve{B}_{j}}\left(u_{k}\right)$, for
all $j, k$, by using Definition 32, we have 


$$
R_{\mathrm{GmPNSS}}\left(F_{\breve{A}^{\prime}}, G_{\breve{B}}\right)=\frac{\sum_{j=1}^{z} \sum_{k=1}^{t}\left(\left(s_{i} \cdot u_{\bar{B}_{j}}\left(u_{k}\right)\right)^{2}+\left(s_{i} \cdot v_{\bar{B}_{j}}\left(u_{k}\right)\right)^{2}+\left(s_{i} \cdot w_{\bar{B}_{j}}\left(u_{k}\right)\right)^{2}\right)}{\sqrt{\sum_{j=1}^{z} \sum_{k=1}^{t}\left(\left(s_{i} \cdot u_{\breve{B}_{j}}\left(u_{k}\right)\right)^{2}+\left(s_{i} \cdot v_{\breve{B}_{j}}\left(u_{k}\right)\right)^{2}+\left(s_{i} \cdot w_{\bar{B}_{j}}\left(u_{k}\right)\right)^{2}\right)} \sqrt{\sum_{j=1}^{z} \sum_{k=1}^{t}\left(\left(s_{i} \cdot u_{\breve{B}_{j}}\left(u_{k}\right)\right)^{2}+\left(s_{i} \cdot v_{\breve{B}_{j}}\left(u_{k}\right)\right)^{2}+\left(s_{i} \cdot w_{\bar{B}_{j}}\left(u_{k}\right)\right)^{2}\right)}} .
$$

Hence, $R_{\text {GmPNSS }}\left(F_{\breve{A}}, G_{\breve{B}}\right)=1$.

Definition 33. Let $F_{\breve{A}}$ and $G_{\breve{B}}$ be two GmPNSS; then the CC between them also can be defined as follows:

$$
\begin{aligned}
& R_{\mathrm{GmPNSS}}^{1}\left(F_{\breve{A}}, G_{\breve{B}}\right)= \frac{\zeta_{\mathrm{GmPNSS}}\left(F_{\breve{A}}, G_{\breve{B}}\right)}{\max \left\{\varepsilon_{\mathrm{GmPNSS}}\left(F_{\breve{A}}, F_{\breve{A}}\right), \varepsilon_{\mathrm{GmPNSS}}\left(G_{\breve{B}}, G_{\breve{B}}\right)\right\}}, \\
& R_{\mathrm{GmPNSS}}^{1}\left(F_{\breve{A}}, G_{\breve{B}}\right)=\frac{\sum_{j=1}^{z} \sum_{k=1}^{t} s_{i} \cdot u_{\breve{A}_{j}}\left(u_{k}\right) s_{i} \cdot u_{\breve{B}_{j}}\left(u_{k}\right)+s_{i} \cdot v_{\breve{A}_{j}}\left(u_{k}\right) s_{i} \cdot v_{\breve{B}_{j}}\left(u_{k}\right)+s_{i} \cdot w_{\breve{A}_{j}}\left(u_{k}\right) s_{i} \cdot w_{\breve{B}_{j}}\left(u_{k}\right)}{\max \left\{\begin{array}{l}
\sum_{j=1}^{z} \sum_{k=1}^{t}\left(\left(s_{i} \cdot u_{\breve{A}_{j}}\left(u_{k}\right)\right)^{2}+\left(s_{i} \cdot v_{\breve{A}_{j}}\left(u_{k}\right)\right)^{2}+\left(s_{i} \cdot w_{\breve{A}_{j}}\left(u_{k}\right)\right)^{2}\right), \\
\sum_{j=1}^{s} \sum_{k=1}^{t}\left(\left(s_{i} \cdot u_{\breve{B}_{j}}\left(u_{k}\right)\right)^{2}+\left(s_{i} \cdot v_{\breve{B}_{j}}\left(u_{k}\right)\right)^{2}+\left(s_{i} \cdot w_{\breve{B}_{j}}\left(u_{k}\right)\right)^{2}\right)
\end{array}\right\}} .
\end{aligned}
$$

Proposition 13. Let $F_{\breve{A}}$ and $G_{\breve{B}}$ are two GmPNSS; then the CC $R_{\text {GmPNSS }}^{1}\left(F_{\breve{A}}, G_{\breve{B}}\right)$ between them satisfies the following properties:

(1) $0 \leq R_{\text {GmPNSS }}^{1}\left(F_{\breve{A}}, G_{\breve{B}}\right) \leq 1$

(2) $R_{G m P N S S}^{1}\left(F_{\breve{A}}, G_{\breve{B}}\right)=R_{G m P N S S}^{1}\left(G_{\breve{B}}, F_{\breve{A}}\right)$

(3) If $F_{\breve{A}}=G_{\breve{B}}$, that is, $s_{i} \cdot u_{\breve{A}_{i}}\left(u_{k}\right)=s_{i} \cdot u_{\breve{B}_{j}}\left(u_{k}\right), s_{i} \cdot v_{\breve{A}}$ $\left(u_{k}\right)^{A}=s_{i} \cdot v_{\widehat{B}_{i}}\left(u_{k}\right)$, and $s_{i} \cdot w_{\breve{A}_{j}}\left(u_{k}\right)=s_{i} \cdot w_{\bar{B}_{j}}\left(u_{k}\right)$, for all $i, j$, where $i \in 1,2,3, \ldots, m$, then ${ }^{B_{j}} R_{G m P N S S}^{1}$ $\left(F_{\breve{A}}, G_{\breve{B}}\right)=1$

Proof. The proof is easy according to Definition 33.

Nowadays, considering that the weight of GmPNSS is very necessary for practical applications, the result of decisions may vary, whenever decision-makers adjust the different weight to every element in the universe of discourse. Consequently, it is particularly significant to plan the weight before decision-making. Let $\dot{\omega}=\left\{\dot{\omega}_{1}, \dot{\omega}_{2}\right.$, $\left.\dot{\omega}_{3}, \ldots, \dot{\omega}_{\mathrm{t}}\right\}$ be a weight vector for experts such as $\dot{\omega}_{\mathrm{k}}>0$ and $\sum_{k=1}^{t} \dot{\omega}_{\mathrm{k}}=1$, and let $\delta=\left\{\delta_{1}, \delta_{2}, \delta_{3}, \ldots, \delta_{n}\right\}$ be a weight vector for parameters such as $\delta_{j}>0$ and $\sum_{j=1}^{z} \delta_{j}=1$. In the following, we develop the WCC between GmPNSS by extending Definitions 32 and 33.

Definition 34. For two GmPNSS $F_{\breve{A}}$ and $G_{\breve{B}}$, the WCC between them can be defined as follows:

$$
\begin{aligned}
& R_{\mathrm{GWMPNSS}}\left(F_{\breve{A}}, G_{\breve{B}}\right)= \frac{\zeta_{\mathrm{GmPNSS}}\left(F_{\breve{A}}, G_{\breve{B}}\right)}{\sqrt{\varepsilon_{\mathrm{GmPNSS}}\left(F_{\breve{A}}, F_{\breve{A}}\right) \varepsilon_{\mathrm{GmPNSS}}\left(G_{\breve{B}}, G_{\breve{B}}\right)}}, \\
& R_{\mathrm{GmPNSS}}\left(F_{\breve{A}}, G_{\breve{B}}\right)=\frac{\sum_{j=1}^{z} \delta_{j}\left(\sum_{k=1}^{t} \dot{\omega}_{k}\left(s_{i} \cdot u_{\breve{A}_{j}}\left(u_{k}\right) s_{i} \cdot u_{\breve{B}_{j}}\left(u_{k}\right)+s_{i} \cdot v_{\breve{A}_{j}}\left(u_{k}\right) s_{i} \cdot v_{\breve{B}_{j}}\left(u_{k}\right)+s_{i} \cdot w_{\breve{A}_{j}}\left(u_{k}\right) s_{i} \cdot w_{\breve{B}_{j}}\left(u_{k}\right)\right)\right)}{\left(\sqrt{\sum_{j=1}^{z} \delta_{j}\left(\sum_{k=1}^{t} \dot{\omega}_{k}\left(\left(s_{i} \cdot u_{\breve{A}_{j}}\left(u_{k}\right)\right)^{2}+\left(s_{i} \cdot v_{\breve{A}_{j}}\left(u_{k}\right)\right)^{2}+\left(s_{i} \cdot w_{\breve{A}_{j}}\left(u_{k}\right)\right)^{2}\right)\right)}\right)} . \\
&\left.\sqrt{\sum_{j=1}^{z} \delta_{j}\left(\sum_{k=1}^{t} \dot{\omega}_{k}\left(\left(s_{i} \cdot u_{\breve{B}_{j}}\left(u_{k}\right)\right)^{2}+\left(s_{i} \cdot v_{\breve{B}_{j}}\left(u_{k}\right)\right)^{2}+\left(s_{i} \cdot w_{\breve{B}_{j}}\left(u_{k}\right)\right)^{2}\right)\right)}\right)
\end{aligned}
$$

Definition 35. Let $F_{\breve{A}}$ and $G_{\breve{B}}$ be two GmPNSS, then the WCC between them can be defined as follows: 


$$
\begin{aligned}
& R_{\mathrm{GWmPNSS}}^{1}\left(F_{\breve{A}}, G_{\breve{B}}\right)=\frac{\zeta_{\mathrm{GmPNSS}}\left(F_{\breve{A}}, G_{\breve{B}}\right)}{\max \left\{\varepsilon_{\mathrm{GmPNSS}}\left(F_{\breve{A}}, F_{\breve{A}}\right), \varepsilon_{\mathrm{GmPNSS}}\left(G_{\breve{B}}, G_{\breve{B}}\right)\right\}}, \\
& R_{\mathrm{GmPNSS}}^{1}\left(F_{\breve{A}}, G_{\breve{B}}\right)=\frac{\sum_{j=1}^{z} \delta_{j}\left(\sum_{k=1}^{t} \dot{\omega}_{k}\left(s_{i} \cdot u_{\bar{A}_{j}}\left(u_{k}\right) s_{i} \cdot u_{\breve{B}_{j}}\left(u_{k}\right)+s_{i} \cdot v_{\breve{A}_{j}}\left(u_{k}\right) s_{i} \cdot v_{\breve{B}_{j}}\left(u_{k}\right)+s_{i} \cdot w_{\breve{A}_{j}}\left(u_{k}\right) s_{i} \cdot w_{\breve{B}_{j}}\left(u_{k}\right)\right)\right)}{\max \left\{\begin{array}{l}
\sum_{j=1}^{z} \delta_{j}\left(\sum_{k=1}^{t} \dot{\omega}_{k}\left(\left(s_{i} \cdot u_{\breve{A}_{j}}\left(u_{k}\right)\right)^{2}+\left(s_{i} \cdot v_{\breve{A}_{j}}\left(u_{k}\right)\right)^{2}+\left(s_{i} \cdot w_{\breve{A}_{j}}\left(u_{k}\right)\right)^{2}\right)\right) \\
\sum_{j=1}^{z} \delta_{j}\left(\sum_{k=1}^{t} \dot{\omega}_{k}\left(\left(s_{i} \cdot u_{\breve{B}_{j}}\left(u_{k}\right)\right)^{2}+\left(s_{i} \cdot v_{\breve{B}_{j}}\left(u_{k}\right)\right)^{2}+\left(s_{i} \cdot w_{\bar{B}_{j}}\left(u_{k}\right)\right)^{2}\right)\right)
\end{array}\right.} .
\end{aligned}
$$

If we consider $\dot{\omega}=\left\{1 / t, t 1 / t n, q \ldots h,_{1 / t\}}\right.$ and $\delta=\{1 /$ $z, t 1 / z n, q \ldots h,_{1 / z\}}$, then $R_{\mathrm{GWmPNSS}} \quad\left(F_{\breve{A}}, G_{\breve{B}}\right)$ and $R_{\mathrm{GWmPNSS}}^{1}\left(F_{\breve{A}}, G_{\breve{B}}\right)$ are reduced to $R_{\mathrm{GWmPNSS}}\left(F_{\breve{A}}, G_{\breve{B}}\right)$ and $R_{\mathrm{GWmPNSS}}^{1}\left(F_{\breve{A}}, G_{\breve{B}}\right)$, respectively, defined in Definitions 32 and 33 .

Proposition 14. Let $F_{\breve{A}}$ and $G_{\breve{B}}$ be two GmPNSS; then the CC $R_{\text {GWmPNSS }}\left(F_{\breve{A}}, G_{\breve{B}}\right)$ between them satisfies the following properties:

(1) $0 \leq R_{G W m P N S S}\left(F_{\breve{A}}, G_{\breve{B}}\right) \leq 1$
(2) $R_{G W m P N S S}\left(F_{\breve{A}}, G_{\breve{B}}\right)=R_{G W m P N S S}\left(G_{\breve{B}}, F_{\breve{A}}\right)$

(3) If $F_{\breve{A}}=G_{\breve{B}}$, that is, $s_{i} \cdot u_{\breve{A}_{j}}\left(u_{k}\right)=s_{i} \cdot u_{\breve{B}_{j}}\left(u_{k}\right)$, $s_{i} \cdot v_{\breve{A}_{j}}\left(u_{k}\right)=s_{i} \cdot v_{\bar{B}_{j}}\left(u_{k}\right)$, $s_{i} \cdot w_{A_{j}}\left(u_{k}\right)=s_{i} \cdot w_{B_{j}}\left(u_{k}\right)$, for all $j, k$, where $i \in 1,2,3, \ldots, m$, then $R_{G W m P N S S}\left(F_{\breve{A}}, G_{\breve{B}}\right)=1$

Proof. $R_{\mathrm{GWmPNSS}}\left(F_{\breve{A}}, G_{\breve{B}}\right) \geq 0$ is trivial, so we just need to prove that $R_{\mathrm{GWmPNSS}}\left(F_{\breve{A}}, G_{\breve{B}}\right) \leq 1$.

We know that

$$
\begin{aligned}
& \zeta_{\mathrm{GWmPNSS}}\left(F_{\breve{A}}, G_{\breve{B}}\right)=\sum_{j=1}^{z} \delta_{j}\left(\sum_{k=1}^{t} \dot{\omega}_{k}\left(s_{i} \cdot u_{\breve{A}_{j}}\left(u_{k}\right) s_{i} \cdot u_{\breve{B}_{j}}\left(u_{k}\right)+s_{i} \cdot v_{\breve{A}_{j}}\left(u_{k}\right) s_{i} \cdot v_{\breve{B}_{j}}\left(u_{k}\right)+s_{i} \cdot w_{\breve{A}_{j}}\left(u_{k}\right) s_{i} \cdot w_{\breve{B}_{j}}\left(u_{k}\right)\right)\right) \\
& =\sum_{j=1}^{z} \delta_{j}\left(\dot{\omega}_{1}\left(s_{i} \cdot u_{\breve{A}_{j}}\left(u_{1}\right) s_{i} \cdot u_{\breve{B}_{j}}\left(u_{1}\right)+s_{i} \cdot v_{\breve{A}_{j}}\left(u_{1}\right) s_{i} \cdot v_{\breve{B}_{j}}\left(u_{1}\right)+s_{i} \cdot w_{\breve{A}_{j}}\left(u_{1}\right) s_{i} \cdot w_{\breve{B}_{j}}\left(u_{1}\right)\right)\right) \\
& +\sum_{j=1}^{z} \delta_{j}\left(\dot{\omega}_{1}\left(s_{i} \cdot u_{\breve{A}_{j}}\left(u_{2}\right) s_{i} \cdot u_{\breve{B}_{j}}\left(u_{2}\right)+s_{i} \cdot v_{\breve{A}_{j}}\left(u_{2}\right) s_{i} \cdot v_{\breve{B}_{j}}\left(u_{2}\right)+s_{i} \cdot w_{\breve{A}_{j}}\left(u_{2}\right) s_{i} \cdot w_{\breve{B}_{j}}\left(u_{2}\right)\right)\right) \\
& + \\
& \text { : } \\
& + \\
& \sum_{j=1}^{z} \delta_{j}\left(\dot{\omega}_{t}\left(s_{i} \cdot u_{\bar{A}_{j}}\left(u_{t}\right) s_{i} \cdot u_{\breve{B}_{j}}\left(u_{t}\right)+s_{i} \cdot v_{\breve{A}_{j}}\left(u_{t}\right) s_{i} \cdot v_{\bar{B}_{j}}\left(u_{t}\right)+s_{i} \cdot w_{\bar{A}_{j}}\left(u_{t}\right) s_{i} \cdot w_{\breve{B}_{j}}\left(u_{t}\right)\right)\right) \\
& =\left\{\begin{array}{c}
\delta_{1}\left(\dot{\omega}_{1}\left(s_{i} \cdot u_{\breve{A}_{1}}\left(u_{1}\right) s_{i} \cdot u_{\breve{B}_{1}}\left(u_{1}\right)+s_{i} \cdot v_{\breve{A}_{1}}\left(u_{1}\right) s_{i} \cdot v_{\bar{B}_{1}}\left(u_{1}\right)+s_{i} \cdot w_{\bar{A}_{1}}\left(u_{1}\right) s_{i} \cdot w_{\bar{B}_{1}}\left(u_{1}\right)\right)\right)+ \\
\delta_{2}\left(\dot{\omega}_{1}\left(s_{i} \cdot u_{\breve{A}_{2}}\left(u_{1}\right) s_{i} \cdot u_{\breve{B}_{2}}\left(u_{1}\right)+s_{i} \cdot v_{\breve{A}_{2}}\left(u_{1}\right) s_{i} \cdot v_{\breve{B}_{2}}\left(u_{1}\right)+s_{i} \cdot w_{\breve{A}_{2}}\left(u_{1}\right) s_{i} \cdot w_{\breve{B}_{2}}\left(u_{1}\right)\right)\right)+ \\
\vdots \\
+ \\
\delta_{z}\left(\dot{\omega}_{1}\left(s_{i} \cdot u_{\breve{A}_{z}}\left(u_{1}\right) s_{i} \cdot u_{\breve{B}_{z}}\left(u_{1}\right)+s_{i} \cdot v_{\breve{A}_{z}}\left(u_{1}\right) s_{i} \cdot v_{\breve{B}_{z}}\left(u_{1}\right)+s_{i} \cdot w_{\breve{A}_{z}}\left(u_{1}\right) s_{i} \cdot w_{\breve{B}_{z}}\left(u_{1}\right)\right)\right)
\end{array}\right\} \\
& +\left\{\begin{array}{c}
\delta_{1}\left(\dot{\omega}_{2}\left(s_{i} \cdot u_{\breve{A}_{1}}\left(u_{2}\right) s_{i} \cdot u_{\breve{B}_{1}}\left(u_{2}\right)+s_{i} \cdot v_{\breve{A}_{1}}\left(u_{2}\right) s_{i} \cdot v_{\breve{B}_{1}}\left(u_{2}\right)+s_{i} \cdot w_{\breve{A}_{1}}\left(u_{2}\right) s_{i} \cdot w_{\breve{B}_{1}}\left(u_{2}\right)\right)\right)+ \\
\delta_{2}\left(\dot{\omega}_{2}\left(s_{i} \cdot u_{\breve{A}_{2}}\left(u_{2}\right) s_{i} \cdot u_{\breve{B}_{2}}\left(u_{2}\right)+s_{i} \cdot v_{\breve{A}_{2}}\left(u_{2}\right) s_{i} \cdot v_{\breve{B}_{2}}\left(u_{2}\right)+s_{i} \cdot w_{\breve{A}_{2}}\left(u_{2}\right) s_{i} \cdot w_{\breve{B}_{2}}\left(u_{2}\right)\right)\right)+ \\
\vdots \\
+ \\
\delta_{z}\left(\dot{\omega}_{2}\left(s_{i} \cdot u_{\breve{A}_{z}}\left(u_{2}\right) s_{i} \cdot u_{\breve{B}_{z}}\left(u_{2}\right)+s_{i} \cdot v_{\breve{A}_{z}}\left(u_{2}\right) s_{i} \cdot v_{\breve{B}_{z}}\left(u_{2}\right)+s_{i} \cdot w_{\breve{A}_{z}}\left(u_{2}\right) s_{i} \cdot w_{\breve{B}_{z}}\left(u_{2}\right)\right)\right)
\end{array}\right\}+
\end{aligned}
$$




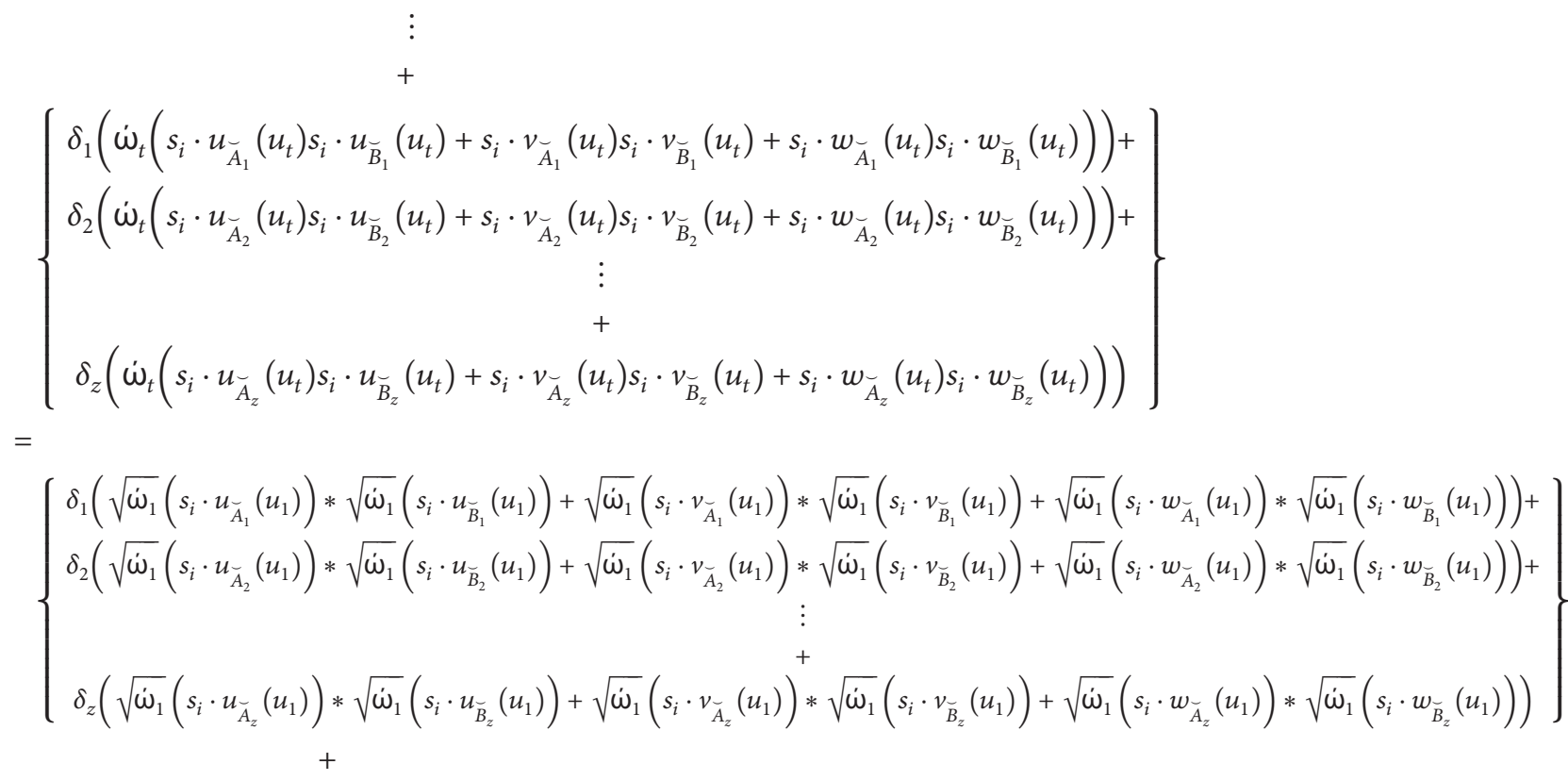

(116)

$$
\begin{aligned}
& \left\{\begin{array}{c}
\delta_{1}\left(\sqrt{\dot{\omega}_{2}}\left(s_{i} \cdot u_{\breve{A}_{1}}\left(u_{2}\right)\right) * \sqrt{\dot{\omega}_{2}}\left(s_{i} \cdot u_{\breve{B}_{1}}\left(u_{2}\right)\right)+\sqrt{\dot{\omega}_{2}}\left(s_{i} \cdot v_{\breve{A}_{1}}\left(u_{2}\right)\right) * \sqrt{\dot{\omega}_{2}}\left(s_{i} \cdot v_{\breve{B}_{1}}\left(u_{2}\right)\right)+\sqrt{\dot{\omega}_{2}}\left(s_{i} \cdot w_{\breve{A}_{1}}\left(u_{2}\right)\right) * \sqrt{\dot{\omega}_{2}}\left(s_{i} \cdot w_{\breve{B}_{1}}\left(u_{2}\right)\right)\right)+ \\
\delta_{2}\left(\sqrt{\dot{\omega}_{2}}\left(s_{i} \cdot u_{\breve{A}_{2}}\left(u_{2}\right)\right) * \sqrt{\dot{\omega}_{2}}\left(s_{i} \cdot u_{\breve{B}_{2}}\left(u_{2}\right)\right)+\sqrt{\dot{\omega}_{2}}\left(s_{i} \cdot v_{\breve{A}_{2}}\left(u_{2}\right)\right) * \sqrt{\dot{\omega_{2}}}\left(s_{i} \cdot v_{\breve{B}_{2}}\left(u_{2}\right)\right)+\sqrt{\dot{\omega}_{2}}\left(s_{i} \cdot w_{\breve{A}_{2}}\left(u_{2}\right)\right) * \sqrt{\dot{\omega}_{2}}\left(s_{i} \cdot w_{\breve{B}_{2}}\left(u_{2}\right)\right)\right)+ \\
\vdots \\
\delta_{z}\left(\sqrt{\dot{\omega}_{2}}\left(s_{i} \cdot u_{\breve{A}_{z}}\left(u_{2}\right)\right) * \sqrt{\dot{\omega}_{2}}\left(s_{i} \cdot u_{\breve{B}_{z}}\left(u_{2}\right)\right)+\sqrt{\dot{\omega}_{2}}\left(s_{i} \cdot v_{\breve{A}_{z}}\left(u_{2}\right)\right) * \sqrt{\dot{\omega}}\left(s_{i} \cdot v_{\breve{B}_{z}}\left(u_{2}\right)\right)+\sqrt{\dot{\omega}_{2}}\left(s_{i} \cdot w_{\breve{A}_{z}}\left(u_{2}\right)\right) * \sqrt{\dot{\omega}_{2}}\left(s_{i} \cdot w_{\breve{B}_{z}}\left(u_{2}\right)\right)\right)
\end{array}\right\} \\
& \text { : } \\
& + \\
& \left\{\begin{array}{c}
\delta_{1}\left(\sqrt{\dot{\omega}_{t}}\left(s_{i} \cdot u_{\breve{A}_{1}}\left(u_{t}\right)\right) * \sqrt{\dot{\omega}_{t}}\left(s_{i} \cdot u_{\breve{B}_{1}}\left(u_{t}\right)\right)+\sqrt{\dot{\omega}_{t}}\left(s_{i} \cdot v_{\breve{A}_{1}}\left(u_{t}\right)\right) * \sqrt{\dot{\omega}_{t}}\left(s_{i} \cdot v_{\breve{B}_{1}}\left(u_{t}\right)\right)+\sqrt{\dot{\omega}_{t}}\left(s_{i} \cdot w_{\breve{A}_{1}}\left(u_{t}\right)\right) * \sqrt{\dot{\omega}_{t}}\left(s_{i} \cdot w_{\breve{B}_{1}}\left(u_{t}\right)\right)\right)+ \\
\delta_{2}\left(\sqrt{\dot{\omega}_{t}}\left(s_{i} \cdot u_{\breve{A}_{2}}\left(u_{t}\right)\right) * \sqrt{\dot{\omega}_{t}}\left(s_{i} \cdot u_{\breve{B}_{2}}\left(u_{t}\right)\right)+\sqrt{\dot{\omega}_{t}}\left(s_{i} \cdot v_{\breve{A}_{2}}\left(u_{t}\right)\right) * \sqrt{\dot{\omega}_{t}}\left(s_{i} \cdot v_{\breve{B}_{2}}\left(u_{t}\right)\right)+\sqrt{\dot{\omega}_{t}}\left(s_{i} \cdot w_{\breve{A}_{2}}\left(u_{t}\right)\right) * \sqrt{\dot{\omega}_{t}}\left(s_{i} \cdot w_{\breve{B}_{2}}\left(u_{t}\right)\right)\right)+ \\
\vdots \\
\delta_{z}\left(\sqrt{\dot{\dot{\omega}_{t}}}\left(s_{i} \cdot u_{\breve{A}_{z}}\left(u_{t}\right)\right) * \sqrt{\dot{\omega}_{t}}\left(s_{i} \cdot u_{\breve{B}_{z}}\left(u_{t}\right)\right)+\sqrt{\dot{\dot{\omega}_{t}}}\left(s_{i} \cdot v_{\breve{A}_{z}}\left(u_{t}\right)\right) * \sqrt{\dot{\dot{\omega}_{t}}}\left(s_{i} \cdot v_{\breve{B}_{z}}\left(u_{t}\right)\right)+\sqrt{\dot{\omega}_{t}}\left(s_{i} \cdot w_{\breve{A}_{z}}\left(u_{t}\right)\right) * \sqrt{\dot{\omega}_{t}}\left(s_{i} \cdot w_{\breve{B}_{z}}\left(u_{t}\right)\right)\right)
\end{array}\right\}
\end{aligned}
$$




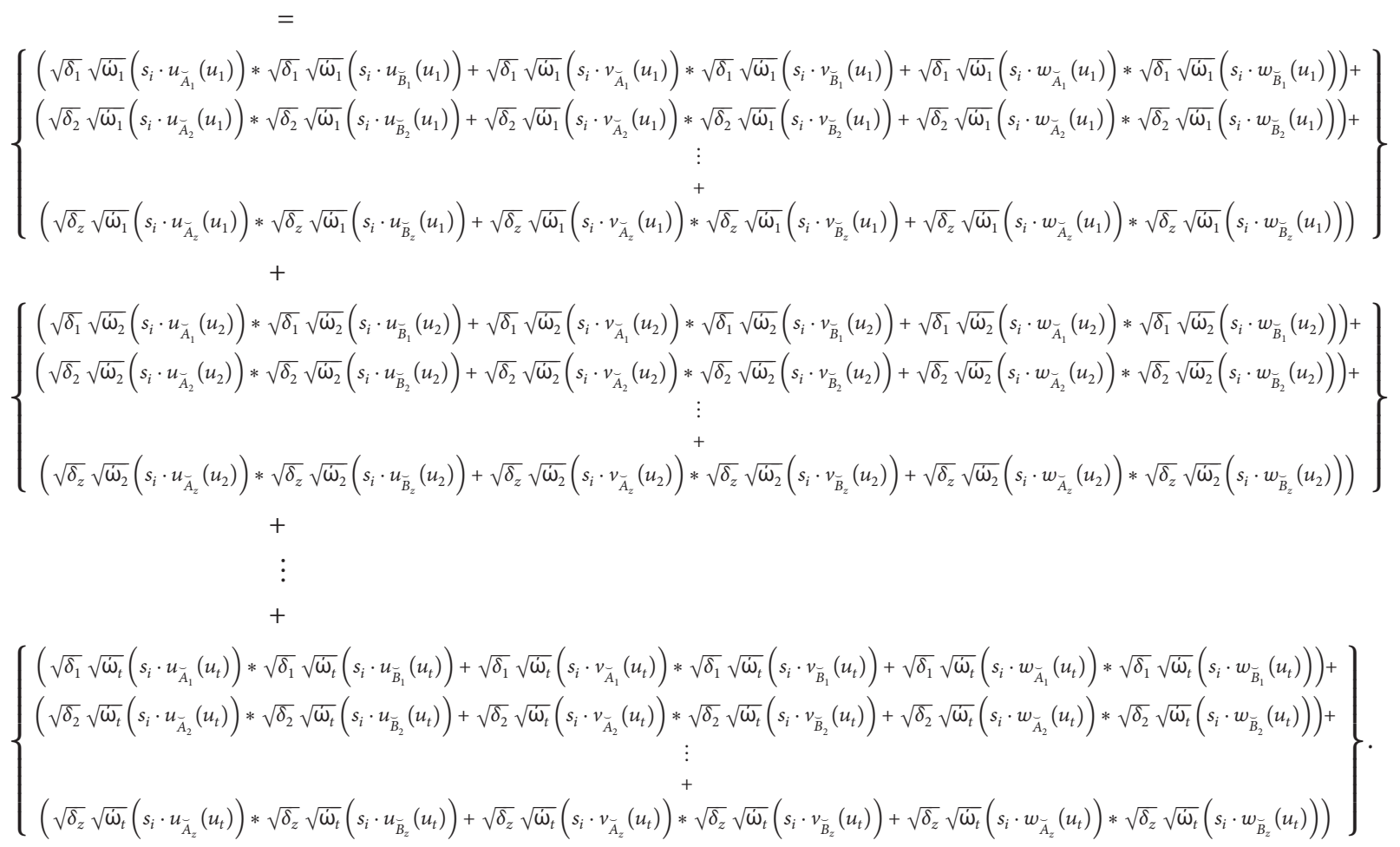

By using Cauchy-Schwarz inequality, we get

$\left(\zeta_{\text {GWmPNSS }}\left(F_{\breve{A}}, G_{\breve{B}}\right)\right)^{2} \leq$

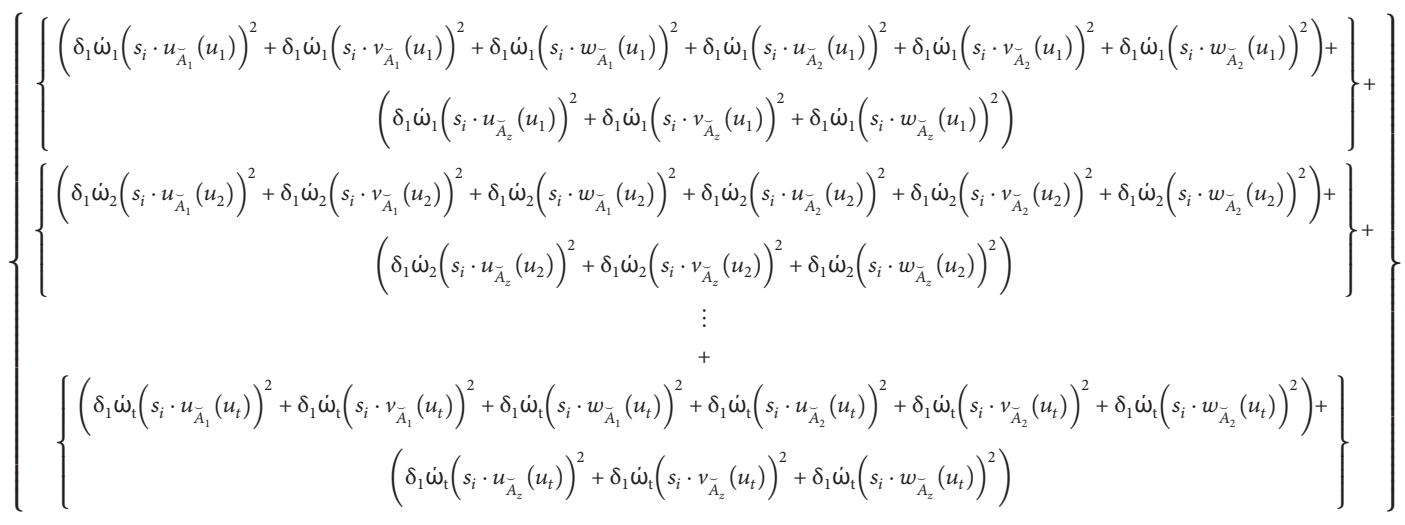


Journal of Mathematics

23

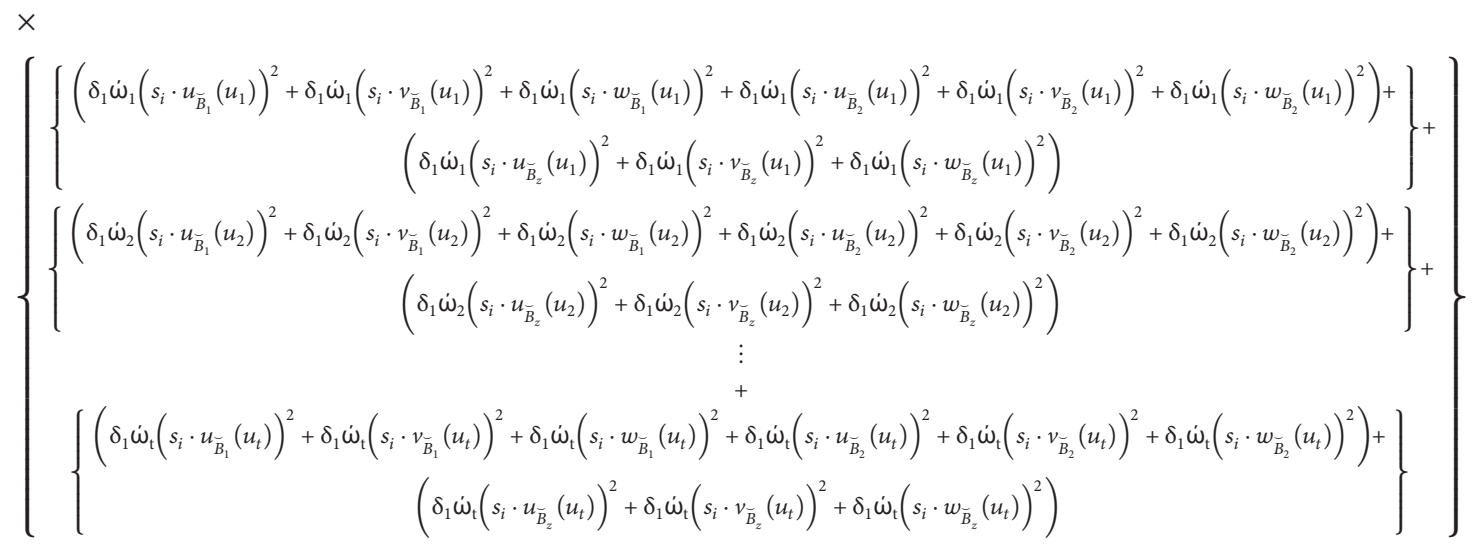

$$
\begin{aligned}
& \left(\zeta_{\mathrm{GWmPNSS}}\left(\mathscr{F}_{\check{A}}, \mathscr{G}_{\check{B}}\right)\right)^{2} \leq \sum_{j=1}^{z} \delta_{j}\left(\sum_{k=1}^{t} \dot{\omega}_{k}\left(\left(s_{i} \bullet u_{\check{A}_{j}}\left(u_{k}\right)\right)^{2}+\left(s_{i} \bullet v_{\check{A}_{j}}\left(u_{k}\right)\right)^{2}+\left(s_{i} \bullet w_{\check{A}_{j}}\left(u_{k}\right)\right)^{2}\right)\right) \\
& \times \sum_{j=1}^{z} \delta_{j}\left(\sum_{k=1}^{t} \dot{\omega}_{k}\left(\left(s_{i} \bullet u_{\check{B}_{j}}\left(u_{k}\right)\right)^{2}+\left(s_{i} \bullet v_{\check{B}_{j}}\left(u_{k}\right)\right)^{2}+\left(s_{i} \bullet w_{\check{B}_{j}}\left(u_{k}\right)\right)^{2}\right)\right) \\
& =\mathscr{E}_{\mathrm{GWmPNSS}}\left(\mathscr{F}_{\check{A}}\right) \cdot \mathscr{E}_{\mathrm{GWmPNSS}}\left(\mathscr{G}_{\check{B}}\right) \text {. }
\end{aligned}
$$

Therefore, $\left(\zeta_{\text {GWmPNSS }}\left(\mathscr{F}_{\check{A}}, \mathscr{G}_{\check{B}}\right)\right)^{2} \leq \mathscr{E}_{\text {GWmPNSS }}\left(\mathscr{F}_{\check{A}}\right)$ $\mathscr{E}_{\mathrm{GWmPNSS}}\left(\mathscr{G}_{\breve{B}}\right)$. Hence, by using Definition 34 , we get $\mathscr{R}_{\mathrm{GWmPNSS}}\left(\mathscr{F}_{\check{A}}, \mathscr{G}_{\check{B}}\right) \leq 1$, so $0 \leq \mathscr{R}_{\mathrm{GWmPNSS}}\left(\mathscr{F}_{\check{A}}, \mathscr{G}_{\check{B}}\right) \leq$ 1.
Proof. The proof is obvious.

Proof. Utilizing Definition 34,

$R_{\mathrm{GWmPNSS}}\left(F_{\breve{A}}, G_{\breve{B}}\right)=$

$$
\frac{\sum_{j=1}^{z} \delta_{j}\left(\sum_{k=1}^{t} \dot{\omega}_{k}\left(s_{i} \cdot u_{\breve{A}_{j}}\left(u_{k}\right) s_{i} \cdot u_{\breve{B}_{j}}\left(u_{k}\right)+s_{i} \cdot v_{\breve{A}_{j}}\left(u_{k}\right) s_{i} \cdot v_{\breve{B}_{j}}\left(u_{k}\right)+s_{i} \cdot w_{\breve{A}_{j}}\left(u_{k}\right) s_{i} \cdot w_{\breve{B}_{j}}\left(u_{k}\right)\right)\right)}{\left(\sqrt{\sum_{j=1}^{z} \delta_{j}\left(\sum_{k=1}^{t} \dot{\omega}_{k}\left(\left(s_{i} \cdot u_{\breve{A}_{j}}\left(u_{k}\right)\right)^{2}+\left(s_{i} \cdot v_{\breve{A}_{j}}\left(u_{k}\right)\right)^{2}+\left(s_{i} \cdot w_{\breve{A}_{j}}\left(u_{k}\right)\right)^{2}\right)\right)}\right)} .
$$

As we know that $s_{i} \bullet u_{\check{A}_{j}}\left(u_{k}\right)=s_{i} \bullet u_{\check{B}_{j}}\left(u_{k}\right)$, $s_{i} \bullet v_{\check{A}_{j}}\left(u_{k}\right)=s_{i} \bullet v_{\check{B}_{j}}\left(u_{k}\right)$, and $s_{i} \bullet w_{\check{A}_{j}}\left(u_{k}\right)=s_{i} \bullet w_{\check{B}_{j}}\left(u_{k}\right)$, for all $j, k$, by using Definition 34 , we have

$$
\mathscr{R}_{\mathrm{GWmPNSS}}\left(\mathscr{F}_{\check{A}}, \mathscr{G}_{\check{B}}\right)=\frac{\sum_{j=1}^{z} \delta_{j}\left(\sum_{k=1}^{t} \dot{\omega}_{k}\left(\left(s_{i} \bullet u_{\check{A}_{j}}\left(u_{k}\right)\right)^{2}+\left(s_{i} \bullet v_{\check{A}_{j}}\left(u_{k}\right)\right)^{2}+\left(s_{i} \bullet w_{\check{A}_{j}}\left(u_{k}\right)\right)^{2}\right)\right)}{\left(\sqrt{\sum_{j=1}^{z} \delta_{j}\left(\sum_{k=1}^{t} \dot{\omega}_{k}\left(\left(s_{i} \bullet u_{\check{A}_{j}}\left(u_{k}\right)\right)^{2}+\left(s_{i} \bullet v_{\check{A}_{j}}\left(u_{k}\right)\right)^{2}+\left(s_{i} \bullet w_{\check{A}_{j}}\left(u_{k}\right)\right)^{2}\right)\right)}\right)} .
$$


Hence, $\mathscr{R}_{\text {GWmPNSS }}\left(\mathscr{F}_{\check{A}}, \mathscr{G}_{\check{B}}\right)=1$.

\section{Applications of Similarity Measures and Correlation Coefficient of GmPNSS in Medical Diagnoses and Decision-Making}

In this section, we proposed the algorithm for GmPNSS by using developed similarity measures and CC. We also used the proposed methods for medical diagnoses and decisionmaking in real-life problems.

6.1. Application of Similarity Measure in Medical Diagnoses. We develop the algorithm of GmPNSS for similarity measure and use the developed similarity measure for medical diagnoses by using the proposed algorithm, shown in Figure 1.

\subsubsection{Algorithm for Similarity Measure of GmPNSS}

Step 1. Pick out the set containing parameters.

Step 2. Construct the GmPNSS according to experts.

Step 3. Construct GmPNSS $\varphi_{\mathscr{G}}^{t}$ for the evaluation of different decision-makers, where $t=1,2, \ldots, m$.

Step 4. Find the distance between two GmPNSS by using the distance formula:

$$
\begin{aligned}
d_{\mathrm{GmPNSS}}^{H}\left(\Phi_{\mathscr{F}}(e), \varphi_{\mathscr{G}}(e)\right)= & \frac{1}{2 m}\left\{\sum_{i=1}^{m} \sum_{j=1}^{p}\left(\left|s_{i} \bullet u_{\Phi_{\mathscr{F}}}\left(u_{j}\right)-s_{i} \bullet u_{\varphi_{\mathscr{G}}}\left(u_{j}\right)\right|\right)+\left(\left|s_{i} \bullet v_{\Phi_{\mathscr{F}}}\left(u_{j}\right)-s_{i} \bullet v_{\varphi_{\mathscr{G}}}\left(u_{j}\right)\right|\right)\right. \\
& \left.+\left(\left|s_{i} \bullet w_{\Phi_{\mathscr{F}}}\left(u_{j}\right)-s_{i} \bullet w_{\varphi_{\mathscr{G}}}\left(u_{j}\right)\right|\right)\right\} .
\end{aligned}
$$

Step 5. Compute the similarity measure between two GmPNSS by utilizing the following formula:

$$
S_{\mathrm{GmPNSS}}\left(\Phi_{\mathscr{F}}, \varphi_{\mathscr{G}}\right)=\frac{1}{1+d\left(\Phi_{\mathscr{F}}, \varphi_{\mathscr{G}}\right)} .
$$

Step 6. Analyze the result.

The flow chart of the presented algorithm can be seen in Figure 1.

6.2. Problem Formulation and Application of Similarity Measure and CC of GmPNSS for Disease Diagnoses. The general proposed algorithm can be used in diagnosis complications. In the light of scientific discipline, a numerical example is presented to deal the diagnostic difficulties. This planned algorithm may be obtained from immoderate medical disease diagnosis complications. We consider typhoid disease as a diagnosis problem, so regardless of whether a well-advised patient has typhoid or not, as many containing the overall signs and symptoms of typhoid are going to be compatible as well as other diseases such as malaria. For a verbal description of the disease, we tend to dispensed similarity measures along the GmPNSS structure to attain an insured person as well as high-fidelity consequences. The general $\mathrm{m}$-polar anatomical structure offers us a record of medical experts rating for the extraordinary disease.

6.2.1. Application of Similarity Measure. Now, we consider the universal set as follows: $\mathscr{U}=\left\{u_{1}=\right.$ typhoid, $u_{2}=$ nontyphoid $\}$ and $E$ is a set of parameters consisting of symptoms of typhoid disease such as $E=\left\{x_{1}=\right.$ flu, $x_{2}=$ body pain, $x_{3}=$ headache $\}$. Consider $\mathscr{F}$ and $\mathscr{G} \subseteq E$; then we construct the G3-PNSS of $\mathscr{F}$ and $\mathscr{G}$ such as $\Phi_{\mathscr{F}}(x)$ and $\varphi_{\mathscr{G}}(x)$ according to experts as given in Tables 1 and 2.

Compute distances between $\Phi_{\mathscr{F}}(x)$ and $\varphi_{\mathscr{G}}(x)$ by using Definition 25 given as follows:

$$
\begin{aligned}
& d_{\mathrm{G} 3-\mathrm{PNSS}}^{\mathrm{H}}\left(\Phi_{\mathscr{F}}(e), \varphi_{\mathscr{G}}(e)\right)=0.6183 \\
& d_{\mathrm{G} 3-\mathrm{NNSS}}^{\mathrm{NH}}\left(\Phi_{\mathscr{F}}(e), \varphi_{\mathscr{G}}(e)\right)=0.3092 \\
& d_{\mathrm{G} 3-\mathrm{PNSS}}^{\mathrm{E}}\left(\Phi_{\mathscr{F}}(e), \varphi_{\mathscr{G}}(e)\right)=0.7749 \\
& d_{\mathrm{G} 3-\mathrm{PNSS}}^{\mathrm{NE}}\left(\Phi_{\mathscr{F}}(e), \varphi_{\mathscr{G}}(e)\right)=0.5481
\end{aligned}
$$

By using Hamming distance, we will find the similarity measure between $\Phi_{\mathscr{F}}(e)$ and $\varphi_{\mathscr{G}}(e)$ given as follows:

$$
S_{\mathrm{G} 3-\mathrm{PNSS}}\left(\Phi_{\mathscr{F}}, \varphi_{\mathscr{G}}\right)=0.6179>0.5 \text {. }
$$

According to the above calculation, $S_{\mathrm{G} 3 \text {-PNSS }}\left(\Phi_{\mathscr{F}}\right.$, $\left.\varphi_{\mathscr{G}}\right) \geq 0.5$, so G3-PNSS of $\Phi_{\mathscr{F}}$ and $\varphi_{\mathscr{G}}$ are significantly similar, which shows that the patient suffers from typhoid.

6.3. Applications of Correlation Coefficient in Medical Diagnoses. We develop the algorithm of GmPNSS for CC and use the developed CC for medical diagnoses by developing an algorithm.

\subsubsection{Algorithm for Correlation Coefficient of GmPNSS}

Step 1. Pick out the set containing parameters.

Step 2. Construct the GmPNSS according to experts.

Step 3. Find the informational neutrosophic energies of any two GmPNSS.

Step 4. Calculate the correlation between two GmPNSS by using the following formula: 
Step 1

(i) Input GmPNSS according to requirement

\section{Step 2}

(ii) Construct the evaluation report for each alternative in the form of GmPNSS

Step 3

(iii) Compute the distance between GmPNSS

Step 4
(iv) Compute the similarity measures

Step 5

(v) Choose the alternative with highest similarity

Step 6

(vi) Analyze the ranking

FIGURE 1: Flow chart of presented algorithm for GmPNSS based on the similarity measure.

TABLE 1: G3-PNSS of $\mathscr{F}_{\check{A}}$ according to experts.

\begin{tabular}{lccc}
\hline$\Phi_{\mathscr{F}}(x)$ & $x_{1}$ & $x_{2}$ & $x_{3}$ \\
\hline$u_{1}$ & $(.69, .52, .61),(.37, .44, .23),(.46, .37, .29)$ & $(.54, .63, .55),(.48, .44, .26),(.63, .47, .59)$ & $(.34, .47, .27),(.46, .48, .37),(.75, .58, .69)$ \\
$u_{2}$ & $(.43, .66, .62),(.48, .45, .53),(.47, .52, .36)$ & $(.17, .23, .29),(.37, .41, .47),(.53, .59, .61)$ & $(.58, .53, .55),(.37, .35, .32),(.65, .63, .59)$ \\
\hline
\end{tabular}

TABLe 2: G3-PNSS of $\mathscr{G}_{\check{\mathrm{B}}}$ according to experts.

\begin{tabular}{cccc}
\hline$\varphi_{\mathscr{G}}(x)$ & $x_{1}$ & $x_{2}$ \\
\hline$u_{1}$ & $(.63, .57, .54),(.47, .46, .32),(.62, .75, .67)$ & $(.45, .71, .50),(.50, .43, .26),(.61, .50, .47)$ & $(.27, .38, .24),(.58, .37, .47),(.65, .69, .70)$ \\
$u_{2}$ & $(.47, .59, .69),(.53, .50, .60),(.43, .58, .32)$ & $(.15, .25, .25),(.32, .40, .43),(.53, .60, .60)$ & $(.47, .46, .64),(.44, .40, .30),(.61, .60, .68)$ \\
\hline & & & \\
& $\zeta_{\text {GmPNSS }}\left(\mathscr{F}_{\check{A}}, \mathscr{G}_{\check{B}}\right)=\sum_{j=1}^{z} \sum_{k=1}^{t}\left(s_{i} \bullet u_{\check{A}_{j}}\left(u_{k}\right) s_{i} \bullet u_{\check{B}_{j}}\left(u_{k}\right)+s_{i} \bullet v_{\check{A}_{j}}\left(u_{k}\right) s_{i} \bullet v_{\check{B}_{j}}\left(u_{k}\right)+s_{i} \bullet w_{\check{A}_{j}}\left(u_{k}\right) s_{i} \bullet w_{\check{B}_{j}}\left(u_{k}\right): i \in 1,2,3, \ldots, m\right)$.
\end{tabular}

Step 5. Calculate the CC between any two GmPNSS by using the following formula:

$$
\mathscr{R}_{\mathrm{GmPNSS}}\left(\mathscr{F}_{\check{A}}, \mathscr{G}_{\check{B}}\right)=\frac{\zeta_{\mathrm{GmPNSS}}\left(\mathscr{F}_{\check{A}}, \mathscr{G}_{\check{B}}\right)}{\sqrt{\mathscr{E}_{\mathrm{GmPNSS}}\left(\mathscr{F}_{\check{A}}, \mathscr{F}_{\check{A}}\right) \cdot \mathscr{E}_{\mathrm{GmPNSS}}\left(\mathscr{G}_{\check{B}}, \mathscr{G}_{\check{B}}\right)}}
$$

Step 6. Analyze the results.

The flow chart of the presented algorithm can be seen in Figure 2.

6.3.2. Application of Correlation Coefficient. We use the proposed algorithm for medical diagnoses. For this, we consider that $\mathscr{F}_{\check{A}}$ and $\mathscr{G}_{\check{B}}$ are G3-PNSS which are described in Section 6.2.1 in Tables 1 and 2, respectively. By using equation (12), we can find CC against the values of the universal set given as follows: $\mathscr{R}_{\mathrm{G} 3-\mathrm{PNSS}\left(u_{1}\right)}\left(\mathscr{F}_{\check{A}}, \mathscr{G}_{\check{B}}\right)=0.9967$ and $\mathscr{R}_{\mathrm{G} 3-\mathrm{PNSS}\left(u_{2}\right)}$ $\left(\mathscr{F}_{\check{A}}, \mathscr{G}_{\check{B}}\right)=0.9925$. By the above calculation, we analyze the results and get $\mathscr{R}_{\mathrm{G} 3-\operatorname{PNSS}\left(u_{1}\right)}\left(\mathscr{F}_{\check{A}}, \mathscr{G}_{\check{B}}\right)>\mathscr{R}_{\mathrm{G} 3-\operatorname{PNSS}\left(u_{2}\right)}\left(\mathscr{F}_{\check{A}}\right.$, $\left.\mathscr{G}_{\check{B}}\right)$, which shows that patient suffers from typhoid. 


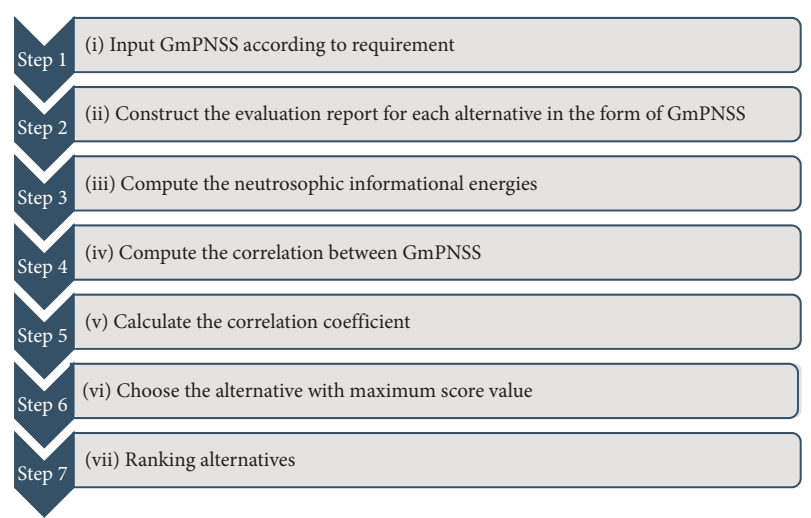

FIgURE 2: Flow chart of the presented algorithm based on the correlation coefficient.

6.4. Problem Formulation and Application of GmPNSS for Decision-Making. Department of the scientific discipline of some university $U$ will have one scholarship for a postdoctoral position. Several applicants apply for scholarship but only four $S=\left\{S_{1}, S_{2}, S_{3}, S_{4}\right\}$ applicants received the interview call for evaluation based on their CGPA (cumulative grade points average). The president of the university hires a committee of four experts $X=\left\{X_{1}, X_{2}, X_{3}, X_{4}\right\}$ for the selection of the postdoctoral scholars. First of all, the committee decides the set of parameters such as $E=\left\{x_{1}, x_{2}\right.$, $\left.x_{3}\right\}$, where $x_{1}, x_{2}$, and $x_{3}$ represent the research papers, research quality, and communication skills for the selection of postdoctoral scholars. The experts evaluate the scholars under defined parameters and forward the performance evaluation to the president of the university. Finally, the president of the university scrutinizes the one best scholar based on the expert's evaluation for the postdoctoral scholarship.

6.4.1. Application of GmPNSS for Decision-Making. Assume that $S=\left\{S_{1}, S_{2}, S_{3}, S_{4}\right\}$ is a set of scholars who are shortlisted for interview and $E=\left\{x_{1}=\right.$ research paper, $x_{2}=$ research quality, $x_{3}=$ interview $\}$ is a set of parameters for the selection of scholarship. Let $\mathscr{F}$ and $\mathscr{G} \subseteq E$; then we construct the G3-PNSS $\Phi_{\mathscr{F}}(x)$ according to the requirement of the scientific discipline department.

Now we will construct the G3-PNSS $\varphi_{\mathscr{G}}^{t}$ according to four experts, where $t=1,2,3,4$.

By using equation (3), we calculate the Euclidean distance between $\Phi_{\mathscr{F}}$ and $\varphi_{\mathscr{G}}^{t}$ as follows:

$$
\begin{aligned}
& d_{\mathrm{G} 3-\mathrm{PNSS}}^{E}\left(\Phi_{\mathscr{F}}, \varphi_{\mathscr{G}}^{1}\right)=1.32 \\
& d_{\mathrm{G} 3-\mathrm{PNSS}}^{E}\left(\Phi_{\mathscr{F}}, \varphi_{\mathscr{G}}^{2}\right)=1.3185 \\
& d_{\mathrm{G} 3-\mathrm{PNSS}}^{E}\left(\Phi_{\mathscr{F}}, \varphi_{\mathscr{G}}^{3}\right)=0.4598 \\
& d_{\mathrm{G} 3-\mathrm{PNSS}}^{E}\left(\Phi_{\mathscr{F}}, \varphi_{\mathscr{G}}^{4}\right)=1.1132
\end{aligned}
$$
follows:

Similarity measures of $\Phi_{\mathscr{F}}$ and $\varphi_{\mathscr{G}}^{t}$ can be calculated as

$$
\begin{aligned}
& S_{\mathrm{G} 3-\mathrm{PNSS}}\left(\Phi_{\mathscr{F}}, \varphi_{\mathscr{G}}^{1}\right)=0.4310 \\
& S_{\mathrm{G} 3-\mathrm{PNSS}}\left(\Phi_{\mathscr{F}}, \varphi_{\mathscr{G}}^{2}\right)=0.4313 \\
& S_{\mathrm{G} 3-\mathrm{PNSS}}\left(\Phi_{\mathscr{F}}, \varphi_{\mathscr{G}}^{3}\right)=0.6850
\end{aligned}
$$

$$
S_{\mathrm{G} 3-\mathrm{PNSS}}\left(\Phi_{\mathscr{F}}, \varphi_{\mathscr{G}}^{4}\right)=0.4732
$$

According to the proposed similarity measure, ranking of the alternatives is $S_{3}>S_{4}>S_{2}>S_{1}$, and it is clear that $S_{\mathrm{G} 3-\mathrm{PNSS}}\left(\Phi_{\mathscr{F}}, \varphi_{\mathscr{G}}^{3}\right)=0.6850>0.5$, which shows that $\Phi_{\mathscr{F}}$ and $\varphi_{\mathscr{G}}^{3}$ are significantly similar to G3-PNSS. So $S_{3}$ is the best scholar for the postdoctoral position. Graphical representation of alternatives ranking can be seen in Figure 3.

6.4.2. Solution by Using Algorithm 2. Now, by using Tables $3-7$, we can find the correlation coefficient for each alternative by using equation (12) given as $\mathscr{R}_{\mathrm{GmPNSS}}$ $\left(\Phi_{\mathscr{F}}, \varphi_{\mathscr{G}}^{1}\right)=.8374, \mathscr{R}_{\mathrm{GmPNSS}}\left(\Phi_{\mathscr{F}}, \varphi_{\mathscr{G}}^{2}\right)=.7821, \mathscr{R}_{\mathrm{GmPNSS}}$ $\left(\Phi_{\mathscr{F}}, \varphi_{\mathscr{G}}^{3}\right)=.9462$, and $\mathscr{R}_{\mathrm{GmPNSS}}\left(\Phi_{\mathscr{F}}, \varphi_{\mathscr{G}}^{4}\right)=.9422$. This shows that $\mathscr{R}_{\mathrm{GmPNSS}}\left(\Phi_{\mathscr{F}}, \varphi_{\mathscr{G}}^{3}\right)>\mathscr{R}_{\mathrm{GmPNSS}}\left(\Phi_{\mathscr{F}}, \varphi_{\mathscr{G}}^{4}\right)>$ $\mathscr{R}_{\mathrm{GmPNSS}}\left(\Phi_{\mathscr{F}}, \varphi_{\mathscr{G}}^{1}\right)>\mathscr{R}_{\mathrm{GmPNSS}}\left(\Phi_{\mathscr{F}}, \varphi_{\mathscr{G}}^{2}\right)$. Hence, $S_{3}$ is the best scholar for a postdoctoral position. In Figure 3, we can see the graphical representation of alternatives ranking.

\section{Result Discussion and Comparative Analysis}

In the following section, we will discuss the effectiveness, naivety, flexibility, and advantages of the proposed methods and algorithms. We also conducted a brief comparative analysis of the following: suggested methods and existing methods.

7.1. Advantages and Flexibility of the Proposed Approach. The recommended technique is effective and applicable to all forms of input data. Here, we introduce two novel algorithms based on GmPNSS: one is CC, and the other is similarity measures. Both algorithms are effective and can provide the best results in MCDM problems. The recommended algorithm is simple and easy to understand, can deepen understanding, and is suitable for many types of choices and indicators. Developed algorithms are flexible and easy to change to suit different situations, inputs, and outputs. There are subtle differences between the rankings of the suggested methods because different techniques have different ranking methods, so they can be afforded according to their considerations.

7.1.1. Superiority of the Proposed Method. Through this research and comparative analysis, we have concluded that the results obtained by the proposed method are more general than the prevailing methods. However, in the decision-making process, compared with the existing decisionmaking methods, it contains more information to deal with the uncertainty in the data. Moreover, the mixed structure of many FS has become a special case of GmPNSS, by adding some suitable conditions. Among them, the information related to the object can be expressed more accurately and empirically, so it is a convenient tool for combining inaccurate and uncertain information in the decision-making process. Therefore, our proposed method is effective, flexible, simple, and superior to other hybrid structures of fuzzy sets. 


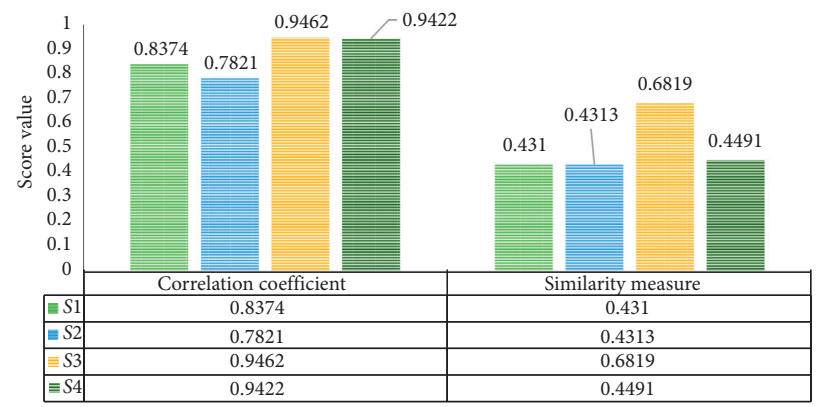

FIgURE 3: Alternative final score value with the proposed algorithms.

TABLE 3: Construction of G3-PNSS of all scholars according to department requirement.

\begin{tabular}{lcccc}
\hline$\Phi_{\mathscr{F}}(x)$ & $x_{1}$ & $x_{2}$ & $x_{3}$ \\
\hline $\mathbf{X}_{1}$ & $(.82, .55, .63),(.55, .46, .28),(.43, .38, .60)$ & $(.43, .68, .86),(.47, .67, .56),(.42, .51, .33)$ & $(.73, .48, .53),(.87, .43, .77),(.76, .53, .62)$ \\
$\mathbf{X}_{2}$ & $(.50, .62, .52),(.93, .57, .80),(.66, .48, .52)$ & $(.77, .54, .81),(.75, .54, .72),(.53, .54, .69)$ & $(.64, .48, .59),(.32, .58, .22),(.94, .64, .62)$ \\
$\mathbf{X}_{3}$ & $(.29, .25, .41),(.73, .34, .32),(.64, .44, .56)$ & $(.36, .45, .27),(.47, .65, .21),(.61, .37, .39)$ & $(.57, .25, .41),(.72, .55, .29),(.64, .31, .34)$ \\
$\mathbf{X}_{4}$ & $(.91, .50, .16),(.30, .24, .63),(.16, .55, .20)$ & $(.69, .52, .61),(.37, .44, .23),(.46, .37, .29)$ & $(.39, .35, .67),(.47, .24, .32),(.40, .71, .56)$ \\
\hline
\end{tabular}

TABLE 4: G3-PNSS evaluation report according to experts of $S_{1}$.

\begin{tabular}{cccc}
\hline$\varphi_{\mathscr{G}}^{1}$ & $x_{1}$ & $x_{2}$ & $x_{3}$ \\
\hline $\mathbf{X}_{1}$ & $(.13, .15, .22),(.89, .78, .83),(.77, .82, .91)$ & $(.91, .50, .16),(.30, .24, .63),(.16, .55, .20)$ & $(.69, .52, .61),(.37, .44, .23),(.46, .37, .29)$ \\
$\mathbf{X}_{2}$ & $(.79, .84, .93),(.36, .18, .26),(.21, .24, .16)$ & $(.39, .35, .67),(.47, .24, .32),(.40, .71, .56)$ & $(.76, .62, .41),(.36, .49, .79),(.53, .59, .91)$ \\
$\mathbf{X}_{3}$ & $(.07, .23, .32),(.12, .18, .20),(.74, .79, .88)$ & $(.70, .22, .11),(.67, .43, .53),(.41, .57, .49)$ & $(.87, .58, .66),(.77, .22, .56),(.57, .33, .29)$ \\
$\mathbf{X}_{4}$ & $(.23, .12, .17),(.25, .16, .22),(.14, .16, .18)$ & $(.74, .62, .66),(.67, .41, .93),(.85, .67, .99)$ & $(.27, .29, .61),(.71, .43, .21),(.47, .70, .89)$ \\
\hline
\end{tabular}

TABLE 5: G3-PNSS evaluation report according to experts of $S_{2}$.

\begin{tabular}{cccc}
\hline$\varphi_{\mathscr{G}}^{2}$ & $x_{1}$ & $x_{2}$ & $x_{3}$ \\
\hline $\mathbf{X}_{1}$ & $(.16, .20, .27),(.83, .87, .89),(70, .75, .86)$ & $(.91, .50, .16),(.30, .24, .63),(.16, .55, .20)$ & $(.69, .52, .61),(.37, .44, .23),(.46, .37, .29)$ \\
$\mathbf{X}_{2}$ & $(.13, .21, .24),(.18, .20, .20),(.70, .84, .90)$ & $(.39, .35, .67),(.47, .24, .32),(.40, .71, .56)$ & $(.76, .62, .41),(.36, .49, .79),(.53, .59, .91)$ \\
$\mathbf{X}_{3}$ & $(.20, .16, .27),(.29, .17, .26),(.14, .15, .12)$ & $(.70, .22, .11),(.67, .43, .53),(.41, .57, .49)$ & $(.87, .58, .66),(.77, .22, .56),(.57, .33, .29)$ \\
$\mathbf{X}_{4}$ & $(.88, .81, .90),(.40, .20, .26),(.22, .27, .17)$ & $(.74, .62, .66),(.67, .41, .93),(.85, .67, .99)$ & $(.27, .29, .61),(.71, .43, .21),(.47, .70, .89)$ \\
\hline
\end{tabular}

TABLE 6: G3-PNSS evaluation report according to experts of $S_{3}$.

\begin{tabular}{cccc}
\hline$\varphi_{\mathscr{G}}^{3}$ & $x_{1}$ & $x_{2}$ & $x_{3}$ \\
\hline $\mathbf{X}_{1}$ & $(.77, .49, .61),(.71, .43, .21),(.47, .40, .69)$ & $(.47, .59, .76),(.67, .62, .56),(.57, .43, .29)$ & $(.70, .54, .61),(.79, .44, .63),(.61, .41, .51)$ \\
$\mathbf{X}_{2}$ & $(.60, .32, .32),(.77, .49, .83),(.76, .32, .59)$ & $(.76, .62, .61),(.56, .49, .79),(.53, .59, .81)$ & $(.69, .62, .67),(.57, .74, .43),(.86, .47, .79)$ \\
$\mathbf{X}_{3}$ & $(.60, .22, .21),(.67, .43, .53),(.49, .57, .49)$ & $(.29, .72, .41),(.30, .66, .29),(.56, .32, .39)$ & $(.74, .52, .66),(.67, .41, .93),(.85, .47, .59)$ \\
$\mathbf{X}_{4}$ & $(.74, .26, .37),(.49, .41, .63),(.44, .35, .32)$ & $(.41, .66, .51),(.39, .27, .36),(.41, .51, .21)$ & $(.60, .16, .47),(.31, .17, .24),(.54, .35, .24)$ \\
\hline
\end{tabular}

TABLE 7: G3-PNSS evaluation report according to experts of $S_{4}$.

\begin{tabular}{ccccc}
\hline$\varphi_{\mathscr{G}}^{4}$ & $x_{1}$ & $x_{2}$ & $x_{3}$ \\
\hline $\mathbf{X}_{1}$ & $(.23, .13, .22),(.31, .25, .43),(.19, .22, .27)$ & $(.43, .68, .86),(.47, .67, .56),(.42, .51, .33)$ & $(.82, .55, .63),(.55, .46, .28),(.43, .38, .60)$ \\
$\mathbf{X}_{2}$ & $(.10, .13, .11),(.91, .84, .69),(.31, .30, .28)$ & $(.27, .29, .61),(.71, .43, .21),(.47, .70, .89)$ & $(.50, .62, .52),(.93, .57, .80),(.66, .48, .52)$ \\
$\mathbf{X}_{3}$ & $(.70, .22, .11),(.67, .43, .53),(.41, .57, .49)$ & $(.70, .22, .11),(.67, .43, .53),(.41, .57, .49)$ & $(.36, .45, .27),(.47, .65, .21),(.61, .37, .39)$ \\
$\mathbf{X}_{4}$ & $(.45, .16, .27),(.91, .67, .23),(.64, .88, .10)$ & $(.67, .81, .17),(.21, .54, .71),(.41, .54, .21)$ & $(.20, .76, .47),(.39, .17, .46),(.41, .53, .22)$ \\
\hline
\end{tabular}


TABLE 8: Comparative analysis between some existing techniques and the proposed approach.

\begin{tabular}{lcccccc}
\hline & Set & Truthiness & Indeterminacy & Falsity & Multipolarity & Loss of information \\
\hline Chen et al. [48] & mPFS & $\checkmark$ & $\times$ & $\times$ & $\checkmark$ & $\times$ \\
Xu et al. [49] & IFS & $\checkmark$ & $\times$ & $\checkmark$ & $\times$ & $\times$ \\
Zhang et al. [50] & IFS & $\checkmark$ & $\checkmark$ & $\checkmark$ & $\times$ & $\checkmark$ \\
Ali et al. [51] & BPNSS & $\checkmark$ & $\checkmark$ & $\checkmark$ & $\checkmark$ & $\times$ \\
Proposed approach & GmPNSS & $\checkmark$ & $\checkmark$ & $\checkmark$ & $\times$ \\
\hline
\end{tabular}

TABle 9: Comparison between GmPNSS and some existing studies.

\begin{tabular}{lcc}
\hline Method & Alternative final ranking & Optimal choice \\
\hline Masooma et al. [16] & $S_{3}>S_{2}>S_{1}>S_{4}$ & $S_{3}$ \\
Saeed et al. [33] & $S_{3}>S_{4}>S_{2}>S_{1}$ & $S_{3}$ \\
Riaz et al. [52] & $S_{3}>S_{2}>S_{1}>S_{4}$ & $S_{3}$ \\
Kamal et al. [22] & $S_{3}>S_{4}>S_{2}>S_{1}$ & $S_{3}$ \\
Proposed algorithm 1 & $S_{3}>S_{4}>S_{2}>S_{1}$ & $S_{3}$ \\
Proposed algorithm 2 & $S_{3}>S_{4}>S_{1}>S_{2}$ & $S_{3}$ \\
\hline
\end{tabular}

It turns out that this is a contemporary issue. Why do we have to express novel algorithms based on the current novel structure? Many indications are compared with other existing methods, and the recommended methods are surely competent. We remember the following fact: the mixed structures have some limitations in IFS, picture fuzzy sets, FS, hesitation fuzzy sets, NS, and other fuzzy sets, so complete information about the situation cannot be provided. But our m-polar model GmPNSS can be the most suitable for MCDM because it can deal with truth, indeterminacy, and falsity. Due to the exaggerated multipolar neutrosophy, these three degrees are independent of each other and provide a lot of information about alternative norms. Other similarity measures of available hybrid structures are converted into special cases of GmPNSS. A comparative analysis of some existing techniques is listed in Table 8. Therefore, compared with intuitionistic, neutrosophy, hesitant, image, and ambiguity substitution, this model is more versatile and can easily resolve complications. The similarity measures established for GmPNSS become better than the existing similarity measures for MCDM.

7.1.2. Discussion. By using the technique of Chen et al. [48], we deal with the multipolar information of fuzzy sets, but, with this method, we cannot deal with the indeterminacy and falsity objects of alternatives. By using the methodologies of Xu et al. [49] and Zhang et al. [50], we cannot deal with the multipolar information and uncertainty part of the alternative. But, on the other hand, the methodology we established involves the truthiness, indeterminacy, and falsity of alternatives with multiple data. Therefore, the technique we developed is more efficient and can provide better results for decision-makers through various information. Ali et al.'s method [51] dealt with the truthiness, indeterminacy, and falsity levels of alternatives, but these techniques cannot manage multiple data. Instead, the method we developed is an advanced technique that can handle alternatives with multiple types of information. It can be seen in Table 8 .
7.1.3. Comparative Analysis. In this article, we propose two types of algorithms. First, an algorithm is proposed based on the correlation coefficient, and the other is based on similarity measures for GmPNSS. Next, both algorithms are utilized to solve practical problems in real life, that is, for the selection of a postdoctoral position. The graphical representation of results obtained by both algorithms is shown in Figure 3. The results show that the proposed technique is effective and practical. Finally, the ranking of all alternatives using the existing methodologies gives the same final decision; that is, the "postdoctoral" position is selected as $S_{3}$. All rankings are also calculated by applying existing methods with the same case study. The proposed method is also compared with other existing methods: Saeed et al. [33], Masooma et al. [16], Riaz et al. [52], and Kamal et al. [22]. The comparison results are listed in Table 9, which shows the final ranking of the top 4 alternatives. It can be observed that the best selections made by the proposed methods are compared with the already established methods which are expressive in themselves and approve the reliability and validity of the proposed method.

\section{Conclusion}

In this paper, we study the mPNSS and propose a generalized version of mPNSS with some basic operations and properties. We also develop the AND operator, OR operator, Truth-Favorite operator, and False-Favorite operator with properties and examples. The concepts of necessity and possibility operations with their properties are developed in this research. The distance-based similarity measures on GmPNSS are established by using the Hamming and Euclidean distances with their properties, and a decisionmaking approach is presented to solve multicriteria decision-making problems. We also established the correlation coefficient and the weighted correlation coefficient of GmPNSS with the decision-making technique. Furthermore, a numerical illustration has been described to solve the MCDM problem by using the proposed decision-making approaches for medical diagnoses and decision-making. A comparative analysis is presented to verify the validity and demonstration of the proposed method. Finally, the suggested techniques showed higher stability and practicality for decision-makers in the decision-making process. Based on the results obtained, it is concluded that the proposed method is most suitable for solving the MCDM problem in today's life. The presented technique is unable to handle the scenario when the information of truth, falsity, and indeterminacy is given in intervals. In the future, the concept of mPNSS will be extended to interval-valued mPNSS and the 
developed techniques to other fields, such as mathematical programming, cluster analysis, and big data analysis.

\section{Data Availability}

No data were used in this manuscript.

\section{Conflicts of Interest}

The authors declare that they have no conflicts of interest.

\section{Acknowledgments}

The author Rifaqat Ali would like to express his gratitude to the Deanship of Scientific Research at King Khalid University, Saudi Arabia, for providing funding research groups (Grant no. R. G. P. 2/71/41).

\section{References}

[1] L. A. Zadeh, "Fuzzy sets," Information and Control, vol. 8, no. 3, pp. 338-353, 1965.

[2] I. B. Turksen, "Interval valued fuzzy sets based on normal forms," Fuzzy Sets and Systems, vol. 20, no. 2, pp. 191-210, 1986.

[3] K. T. Atanassov, "Intuitionistic fuzzy sets," Fuzzy Sets and Systems, vol. 20, no. 1, pp. 87-96, 1986.

[4] F. Smarandache, "Neutrosophic set-a generalization of intuitionistic fuzzy sets," International Journal of Pure and Applied Mathematics, vol. 24, no. 3, pp. 287-297, 2005.

[5] D. Molodtsov, "Soft set theory-First results," Computers \& Mathematics with Applications, vol. 37, no. 4-5, pp. 19-31, 1999.

[6] P. K. Maji, R. Biswas, and A. R. Roy, "Soft set theory," Computers \& Mathematics with Applications, vol. 45, no. 4-5, pp. 555-562, 2003.

[7] P. K. Maji, A. R. Roy, and R. Biswas, "An application of soft sets in A decision making problem," Computers \& Mathematics with Applications, vol. 44, no. 8-9, pp. 1077-1083, 2002.

[8] M. I. Ali, F. Feng, X. Liu, W. K. Min, and M. Shabir, "On some new operations in soft set theory," Computers \& Mathematics with Applications, vol. 57, no. 9, pp. 1547-1553, 2009.

[9] A. Sezgin and A. O. Atagün, "On operations of soft sets," Computers \& Mathematics with Applications, vol. 61, no. 5, pp. 1457-1467, 2011.

[10] P. K. Maji, "Neutrosophic soft set," Annals of Fuzzy Mathematics and Informatics, vol. 5, no. 1, pp. 157-168, 2013.

[11] F. Karaaslan, "Possibility neutrosophic soft sets and PNSdecision making method," Applied Soft Computing Journal, vol. 54, pp. 403-414, 2016.

[12] S. Broumi, "Generalized neutrosophic soft set," International Journal of Computer Science, Engineering and Information Technology, vol. 3, no. 2, pp. 17-30, 2013.

[13] I. Deli and Y. Şubaş, "A ranking method of single valued neutrosophic numbers and its applications to multi-attribute decision making problems," International Journal of Machine Learning and Cybernetics, vol. 8, no. 4, pp. 1309-1322, 2017.

[14] H. Wang, F. Smarandache, and Y. Zhang, "Single valued neutrosophic sets," International Journal of Machine Learning and Cybernetics, vol. 42, pp. 386-394, 2013.

[15] J. Ye, "A multicriteria decision-making method using aggregation operators for simplified neutrosophic sets," Journal of Intelligent \& Fuzzy Systems, vol. 26, no. 5, pp. 2459-2466, 2014.

[16] M. R. Hashmi, M. Riaz, and F. Smarandache, "m-Polar neutrosophic topology with applications to multi criteria decision-making in medical diagnosis and clustering analysis," International Journal of Machine Learning and Cybernetics, vol. 2019, 2019.

[17] P. P. Dey, S. Pramanik, and B. C. Giri, "Neutrosophic soft multi-attribute decision making based on grey relational projection method," Neutrosophic Sets and Systems, vol. 11, pp. 98-106, 2016.

[18] S. Pramanik, S. Dalapati, S. Alam, and T. K. Roy, "VIKOR based MAGDM strategy under BipolarNeutrosophic set environment," Neutrosophic Sets and Systems, vol. 19, pp. 57-69, 2018.

[19] S. Pramanik, P. P. Dey, and B. C. Giri, "TOPSIS for single valued neutrosophic soft expert SetBased multi-attribute decision making problems," Neutrosophic Sets and Systems, vol. 10, pp. 88-95, 2015.

[20] S. Pramanik, P. P. Dey, B. C. Giri, and F. Smarandache, "Bipolar neutrosophic projection based models forSolving multi-attribute decision making problems," Neutrosophic Sets and Systems, vol. 15, pp. 70-79, 2017.

[21] H.-g. Peng, H.-y. Zhang, and J.-q. Wang, "Probability multivalued neutrosophic sets and its application in multi-criteria group decision-making problems," Neural Computing and Applications, vol. 30, no. 2, p. 563, 2016.

[22] M. Kamal, N. Liyana, and L. Abdullah, "Multi-valued neutrosophic soft set, Malaysian," Journal of Mathematical Sciences, vol. 13, pp. 153-168, 2019.

[23] H. Garg, "An improved cosine similarity measure for intuitionistic fuzzy sets and their applications to decision-making process," Hacettepe Journal of Mathematics and Statistics, vol. 47, no. 6, pp. 1578-1594, 2018.

[24] H. Garg and K. Kumar, "An advanced study on the similarity measures of intuitionistic fuzzy sets based on the set pair analysis theory and their application in decision making," Soft Computing, vol. 47, 2018.

[25] H. Garg and D. Rani, "A robust correlation coefficient measure of complex intuitionistic fuzzy sets and their applications in decision-making," Applied Intelligence, vol. 47, 2018.

[26] H. Garg, "A novel correlation coefficients between pythagorean fuzzy sets and its applications to decision-making processes," International Journal of Intelligent Systems, vol. 10, pp. 1-19, 2016.

[27] R. M. Zulqarnain, X. L. Xin, H. Garg, and W. A. Khan, "Aggregation operators of pythagorean fuzzy soft sets with their application for green supplier chain management," Journal of Intelligent \& Fuzzy Systems, vol. 40, no. 3, 2021.

[28] R. M. Zulqarnain, X. L. Xin, I. Siddique, W. Asghar Khan, and M. A. Yousif, "TOPSIS method based on correlation coefficient under pythagorean fuzzy soft environment and its application towards green supply chain management," Sustainability, vol. 13, no. 4, p. 1642, 2021.

[29] X. T. Nguyen, V. D. Nguyen, V. H. Nguyen, and H. Garg, "Exponential similarity measures for Pythagorean fuzzy sets and their applications to pattern recognition and decisionmaking process," Complex \& Intelligent Systems, vol. 5, no. 2, pp. 217-228, 2019.

[30] X. Peng and H. Garg, "Multiparametric similarity measures on pythagorean fuzzy sets with applications to pattern recognition," Applied Intelligence, vol. 23, 2019. 
[31] L. Wang and N. Li, "Pythagorean fuzzy interaction power Bonferroni mean aggregation operators in multiple attribute decision making," International Journal of Intelligent Systems, vol. 35, no. 1, pp. 50-183, 2020.

[32] L. Wang, H. Garg, and N. Li, "Pythagorean fuzzy interactive Hamacher power aggregation operators for assessment of express service quality with entropy weight," Soft Computing, vol. 25, no. 2, pp. 973-993, 2021.

[33] M. Saeed, M. Saqlain, A. Mehmood, K. Naseer, and S. Yaqoob, "Multi-polar neutrosophic soft sets with application in medical diagnosis and decision-making," Neutrosophic Sets and Systems, vol. 33, pp. 183-207, 2020.

[34] T. Gerstenkorn and J. Mańko, "Correlation of intuitionistic fuzzy sets,” Fuzzy Sets and Systems, vol. 44, no. 1, pp. 39-43, 1991.

[35] C. Yu, "Correlation of fuzzy numbers," Fuzzy Sets and Systems, vol. 55, no. 3, pp. 303-307, 1993.

[36] D.-A. Chiang and N. P. Lin, "Correlation of fuzzy sets," Fuzzy Sets and Systems, vol. 102, no. 2, pp. 221-226, 1999.

[37] W.-L. Hung and J.-W. Wu, "Correlation of intuitionistic fuzzy sets by centroid method," Information Sciences, vol. 144, no. 1-4, pp. 219-225, 2002.

[38] D. H. Hong, "A note on correlation of interval-valued intuitionistic fuzzy sets," Fuzzy Sets and Systems, vol. 95, no. 1, pp. 113-117, 1998.

[39] H. B. Mitchell, "A correlation coefficient for intuitionistic fuzzy sets," International Journal of Intelligent Systems, vol. 19, no. 5, pp. 483-490, 2004.

[40] J. Ye, "Multicriteria decision-making method using the correlation coefficient under single-valued neutrosophic environment," International Journal of General Systems, vol. 42, no. 4, pp. 384-394, 2013.

[41] H. Xue, M. Yu, and C. Chen, "Research on novel correlation coefficient of neutrosophic cubic sets and its applications," Mathematical Problems in Engineering, vol. 2019, 2019.

[42] R. M. Zulqarnain, X. L. Xin, M. Saeed, F. Smarandache, and N. Ahmad, "Generalized neutrosophic TOPSIS to solve multicriteria decision-making problems," Neutrosophic Sets and Systems, vol. 38, pp. 276-292, 2020.

[43] H. Garg, R. Arora, and R. Arora, "TOPSIS method based on correlation coefficient for solving decision-making problems with intuitionistic fuzzy soft set information," AIMS Mathematics, vol. 5, no. 4, pp. 2944-2966, 2020.

[44] S. Iryna, Y. Zhong, W. Jiang, X. Deng, and J. Geng, "Singlevalued neutrosophic set correlation coefficient and its application in fault diagnosis," Symmetry, vol. 12, no. 8, 2020.

[45] F. Karaaslan, "Correlation coefficient between possibility neutrosophic soft sets," Mathematical Sciences Letters, vol. 5, no. 1, pp. 71-74, 2016.

[46] F. Karaaslan, "Correlation coefficients of single-valued neutrosophic refined soft sets and their applications in clustering analysis," Neural Computing and Applications, vol. 28, no. 9, 2016.

[47] S. Broumi and I. Deli, "Correlation measure for neutrosophic refined sets and its application in medical diagnosis," Palestine Journal of Mathematics, vol. 5, no. 1, pp. 135-143, 2016.

[48] J. Chen, S. Li, S. Ma, and X. Wang, "m-Polar fuzzy sets: an extension of bipolar fuzzy sets," Decision Making, vol. 2014, 2014.

[49] Z. Xu, J. Chen, and J. Wu, "Clustering algorithm for intuitionistic fuzzy sets," Information Sciences, vol. 178, no. 19, pp. 3775-3790, 2008.
[50] H. M. Zhang, Z. S. Xu, and Q. Chen, "On clustering approach to intuitionstic fuzzy sets," Information Sciences, vol. 22, pp. 882-888, 2007.

[51] M. Ali and N. D. Tien, "Bipolar neutrosophic soft sets and applications in decision making," Journal of Intelligent \& Fuzzy Systems, vol. 33, no. 6, pp. 4077-4087, 2017.

[52] M. Riaz, N. Khalid, I. Zareef, and D. Afzal, "Neutrosophic $N$ soft sets with TOPSIS method for multiple attribute," Decision Making, vol. 32, pp. 1-24, 2020. 